\title{
Interaction between torsional and lateral vibrations in flexible rotor systems with discontinuous friction
}

\author{
N. Mihajlović • N. van de Wouw • \\ P. C. J. N. Rosielle $\cdot$ H. Nijmeijer
}

Received: 27 April 2006 / Accepted: 26 July 2006 / Published online: 16 January 2007

(C) Springer Science + Business Media B.V. 2007

\begin{abstract}
In this paper, we analyze the interaction between friction-induced vibrations and self-sustained lateral vibrations caused by a mass-unbalance in an experimental rotor dynamic setup. This study is performed on the level of both numerical and experimental bifurcation analyses. Numerical analyses show that two types of torsional vibrations can appear: frictioninduced torsional vibrations and torsional vibrations due to the coupling between torsional and lateral dynamics in the system. Moreover, both the numerical and experimental results show that a higher level of mass-unbalance, which generally increases the lateral vibrations, can have a stabilizing effect on the torsional dynamics, i.e. friction-induced limit cycling can disappear. Both types of analysis provide insight in the fundamental mechanisms causing self-sustained oscillations in rotor systems with flexibility, mass-unbalance and discontinuous friction which support the design of such flexible rotor systems.
\end{abstract}

Present address: N. Mihajlović, Philips Research Laboratories, High Tech Campus 34 (WB21), 5656 AE Eindhoven, The Netherlands

N. Mihajlović $(\bowtie)$. N. van de Wouw · P. C. J. N. Rosielle · H. Nijmeijer

Department of Mechanical Engineering, Eindhoven University of Technology, P.O. Box 513, $5600 \mathrm{MB}$

Eindhoven, The Netherlands

e-mail: Nenad.Mihajlovic@philips.com
Keywords Flexible rotor systems - Discontinuous bifurcations · Experimental non-smooth dynamics . Interaction between torsional and lateral vibrations

\section{Introduction}

Rotating machinery such as turbines, pumps and fans are very important components in many machines and systems. Some examples are aircraft engines, power stations, large flywheels in hybrid transmissions of cars, etc. The behaviour of these rotor-dynamic components can influence the performance of the system as a whole. Namely, for certain ranges of the rotational speed, such systems can exhibit various types of vibration which can be so violent that they can cause significant damage or be performance limiting factors.

There are many causes for such behaviour. Some examples are friction or fluid forces in the bearings in which a shaft is borne, mass-unbalance in a rotor which can lead to whirling motions, flexibilities present in a system, etc. Consequently, the dynamic behaviour of such systems can be very complex (see, for example $[15,23,30,31,32])$.

Krauter [13] analyzed torsional vibrations in water lubricated bearings and authors in [2, 3, 16, 21, 24, 25] analyzed torsional vibrations in drill-string systems. In those papers, it is concluded that torsional vibrations are caused by negative damping in the friction force in the bearings [13], the friction force at the contact between the bit and the borehole rotor [2, 3, 16, 21] and by the friction force at the rotor $[24,25]$. 
Lateral vibrations in rotor systems have been analyzed extensively in $[7,8,15,23,26,30]$. In the work of Lee [15], Tondl [30] and Mihajlović [23], different types of rotor systems are considered; however, in all those systems, lateral vibrations are induced by the mass-unbalance in the rotor. On the other hand, Fritz $[7,8]$ and Muszynska [26] derived expressions for fluid forces which can also induce lateral vibrations in systems with a long vibrating rotor which rotates in a stator.

Interaction between torsional and lateral vibrations in different rotor systems is studied in $[11,15,23,30$, 32]. In various mechanical systems, it is noticed that the increase of the mass-unbalance can have both stabilizing and destabilizing effects in the considered system. For example, Tondl [30], Lee [15] and Mihajlović [23] consider a simple disc with a mass-unbalance connected to a shaft which is elastic in both torsional and lateral directions. They noticed that in such systems, under certain conditions, instabilities can appear if the unbalance increases. However, in [11, 32], the opposite effect has been noticed. Namely, the behaviour of flexible rotor-bearing systems is analyzed and it is concluded that the mass-unbalance can stabilize some rotor systems.

In this paper, we focus on interaction between friction-induced torsional vibrations in flexible mechanical systems and lateral vibrations in rotor systems caused by a mass-unbalance. When analyzing the friction-induced vibrations, a discontinuous static model for the friction is used. We choose such a model and not a more complicated dynamical friction model since it accounts for several essential friction characteristics but avoids the inclusion of unnecessary complexity. A discontinuous friction model leads to a discontinuous model of the system dynamics which exhibits both friction-induced vibrations and the interaction between friction-induced vibrations and vibrations due to mass-unbalance. The discontinuity will have significant consequences for the analysis of the steady-state behaviour of the system. The occurrence, prediction and analysis of limit-cycling behaviour (vibrations) in systems with discontinuities is currently receiving wide attention (see, e.g. [4-6, 9, 14, 16-20, 28]) However, most authors are studying such systems from a theoretical point of view.

Therefore, the focus of this paper is on an experimental study of such system. For this purpose, we constructed an experimental setup. This setup was inspired by a real drilling system which is used for the exploration of oil and gas and which undergoes several types of vibrations during drilling. The setup consists of a DC-motor which is connected to a disc (the upper disc) via a gear box. The upper disc is connected via a low-stiffness string to another disc (the lower disc), and at the lower disc, an additional brake is used. The lower disc can rotate around its geometric centre and can also move in lateral direction. Consequently, such system can undergo torsional vibrations induced by the friction, lateral vibrations due to an additional massunbalance which can be established at the lower disc and a combination of those two types of vibrations. Moreover, the sticking phenomenon is observed due to the presence of the friction, and therefore, a discontinuous nonlinearity (in the friction) plays a crucial role in the dynamical behaviour of the system. Using this setup, the obtained analytical results (on the nonlinear dynamics and (nonsmooth) bifurcations) can be validated experimentally.

In Section 2, the setup is described and the model is given. The dynamic model is described by differential equations with discontinuous right-hand side (due to the presence of friction). Then, the parameters of the model are estimated. In Section 3, the system dynamics are analyzed. The focus of the analysis is on the steady-state behaviour of the system when a constant input voltage is applied at the motor. Consequently, torsional vibrations, lateral vibrations and the interaction between those vibrations in flexible rotor systems with discontinuous friction are modelled and analyzed. As a result of such analysis, appropriate bifurcation diagrams are constructed. This analysis is aiming at an improved understanding of, firstly, the cause of frictioninduced limit cycling and effects of interaction between the torsional and lateral dynamics that influence this type of limit cycling, and secondly, limit cycling induced by the interaction itself. In Section 4, we compare bifurcation diagrams based on the estimated model to experimentally obtained bifurcation diagrams. We also discuss the experimental results obtained for various mass-unbalance levels present at the lower disc. Finally, conclusions are presented.

\section{The experimental setup}

\subsection{Description of the setup}

The experimental setup is shown in Fig. 1. The setup consists of a power amplifier, a DC-motor, two 
Fig. 1 Experimental setup

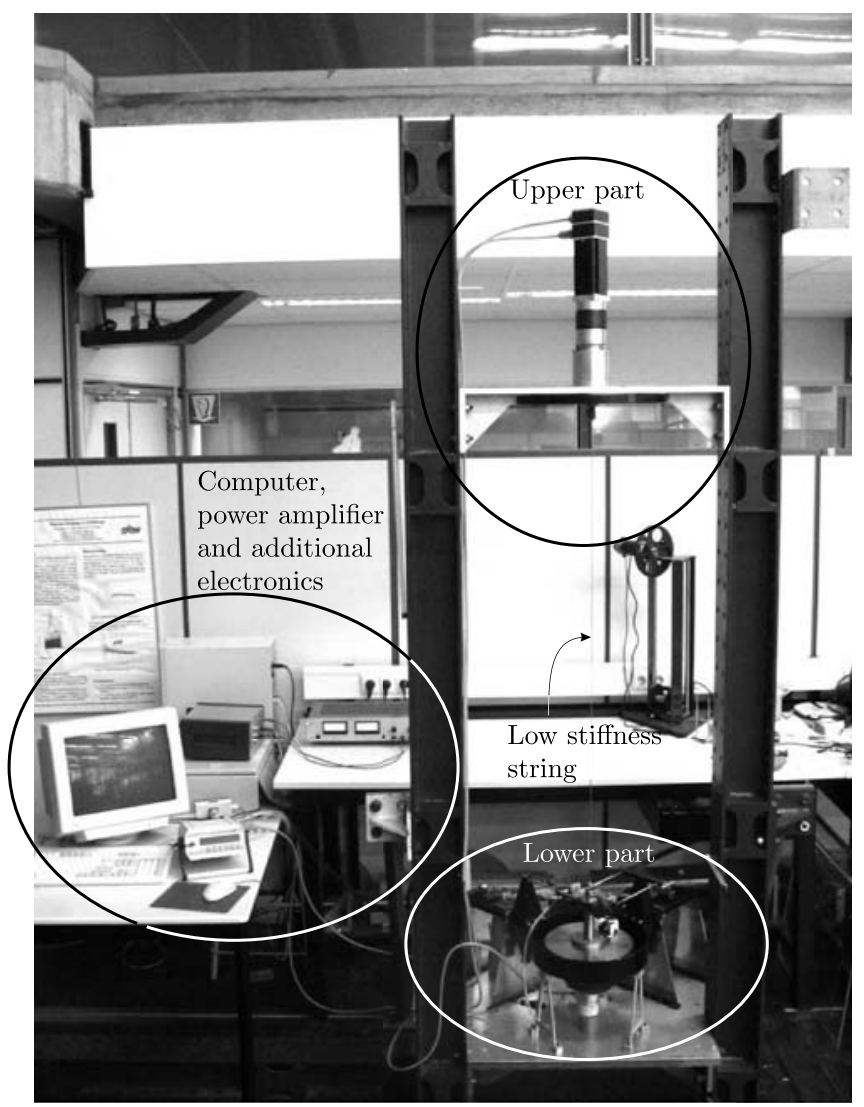

(a) Photo.

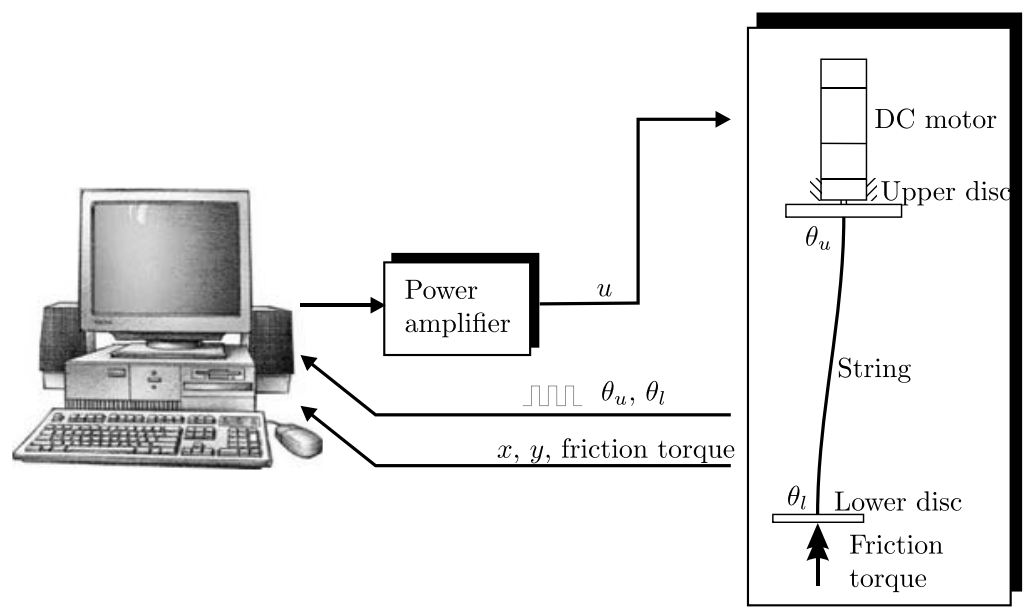

(b) Schematic representation.

rotational (upper and lower) discs, a low-stiffness string and an additional brake applied to the lower disc. The input voltage from the computer ( $u$ in Fig. 1b) is fed into the DC-motor via the power amplifier. The motor is connected, via the gear box, to the upper steel disc. The upper disc and the lower disc are connected through a low-stiffness steel string. The lower disc can rotate around its geometric centre and is also free to move in lateral directions. 
Fig. 2 The lower part of the experimental setup

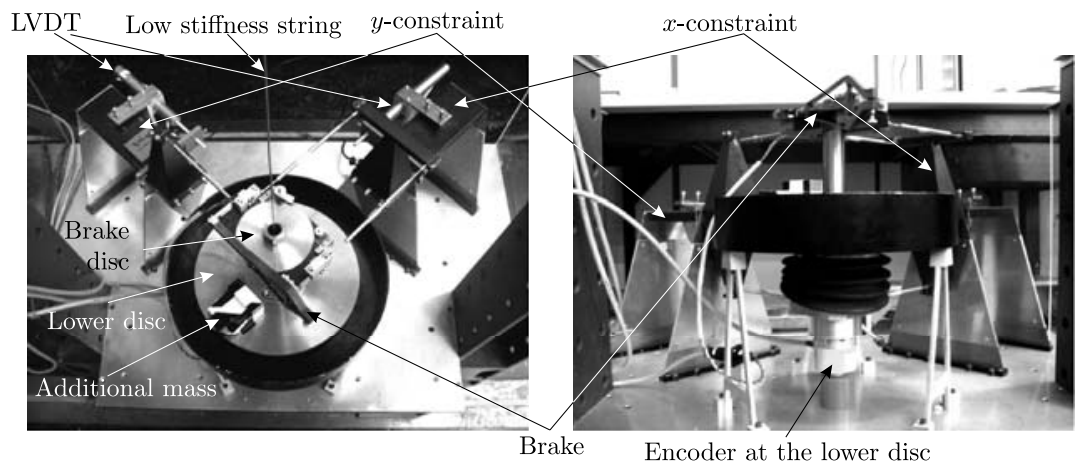

(a) A top view

(b) A front view
In order to induce torsional vibrations at the lower disc, a brake and a small oil-box with felt stripes are fixed to the upper bearing housing of the lower part of the setup (see Fig. 2). With the brake, a range of normal forces can be applied and the contact between the brake and the brake disc produces a friction force exerted on the brake disc. The brake contact material is bronze. The steel brake disc is connected to the lower brass disc via a very stiff shaft. The oil-box with the felt stripes is constructed in order to add oil to the brake disc in a reproducible way. Namely, when some liquid is present in the box, the liquid can be added to both sides of the brake disc due to the capillary effect of the felt. This oil lubrication will prove to be crucial for the existence of torsional vibrations in the setup.

Lateral vibrations are induced by fixing an additional mass at the lower brass disc (Fig. 2). Consequently, a mass-unbalance is introduced to the disc which leads to motions in the lateral plane (whirl-type motion). However, to limit the complexity of the system, tilting of the lower disc is avoided by means of two constraints; one in $x$ - and one in $y$-direction. The constraint in $x$ direction consists of three flexible rods, a rigid body and two leaf springs. Two upper flexible rods are, at one end, connected to the upper bearing housing, and at the other end, to the rigid body of the constraint. These (upper) two rods suppress rotation of the upper bearing housing around the vertical axis; note, in this respect, that the brake is implemented at the bearing housing. The third (lower) flexible rod connects the lower bearing housing to the rigid body of the constraint. The combination of these rods suppresses tilting of the lower disc around $y$-axis. The rigid body of the constraint is connected to the fixed world via two leaf-springs, which allow movement in $x$-direction. The construction of the constraint in $y$-direction is similar, except that only two flexible rods are implemented here: one fixed to the upper bearing housing and other to the lower bearing housing of the lower part. These rods suppress tilting of the lower disc around the $x$-axis.

Both constraints can be fixed using appropriate mechanisms, and when effected, the lower disc cannot move in lateral direction but can only rotate around its geometric centre. In such a way, an experimental setup is created, which can only undergo torsional vibrations (by fixing the constraints and applying the brake), only lateral vibrations (by releasing the constraints and without applying any normal force at the brake) and a combination of those two types of vibrations (by releasing the constraints and applying the brake).

The angular positions of the upper and lower disc $\left(\theta_{u}\right.$ and $\theta_{l}$, respectively, in Fig. $\left.1 \mathrm{~b}\right)$ are measured using incremental encoders. The angular velocities of both discs are obtained by numerical differentiation of the angular positions and filtering the resulting signals using a low-pass filter with a cut-off frequency of $200 \mathrm{rad} / \mathrm{s}(31.8 \mathrm{~Hz})$. The displacements of the geometric centre of the lower disc in $x$ - and $y$-directions are measured with two linear variable differential transformer (LVDT) displacement sensors. The displacement sensors measure, in fact, the displacements of the rigid bodies of the constraints in $x$ - and $y$-directions which equal the displacement of the lower disc in those two directions.

\subsection{Model of the setup}

In order to derive a model of the setup, the EulerLagrange equations are used and the eighth-order system is obtained. The model of the setup is derived in the co-rotating coordinate frame which is fixed to the upper disc as shown in Fig. 3b. The main reason for 
Fig. 3 The drill-string system

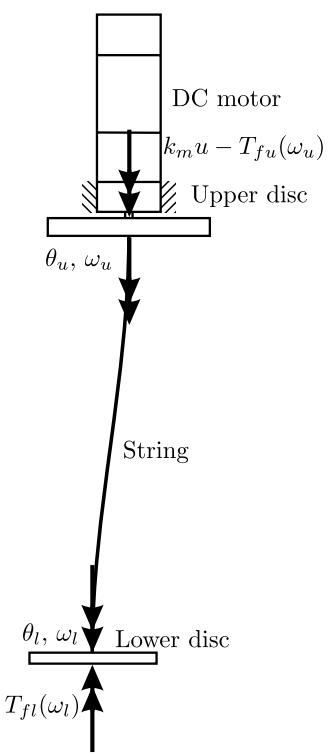

(a) The model of the setup.

adopting a co-rotating coordinate frame is the following: when both the upper and lower discs rotate with constant velocity, a forward whirling motion is performed by the lower disc (lateral vibrations), i.e. the centre of the lower disc moves in the same direction as the rotation of the disc, and this represents a periodic solution in the fixed coordinate frame. The period time of such periodic solution corresponds to the angular velocity of the discs. Moreover, when torsional vibrations appear in the system, then the ratio between period time of torsional vibrations and of whirling motion is, in general, an irrational number. Therefore, such motion represents a quasi-periodic motion in the fixed coordinate frame. However, in the co-rotating coordinate frame, in terms of which the model is formulated, the whirling motion represents an equilibrium point. Moreover, in terms of the co-rotating coordinate frame, the quasi-periodic solution, in the case of fixed coordinates, appears as a periodic solution.

The dynamics of the setup is independent of the angular position of the discs $\left(\theta_{u}\right.$ and $\left.\theta_{l}\right)$, but only depends on the difference $\alpha=\theta_{l}-\theta_{u}$ between these two angular positions (see Fig. 3). Therefore, we replace $\dot{\theta}_{u}$ with $\omega_{u}$, and after performing some equivalent transformations to the obtained dynamic model, the following seventh-order system of differential equations can be obtained:

$$
\begin{aligned}
& J_{u} \dot{\omega}_{u}-k_{\theta} \alpha+T_{f u}\left(\omega_{u}\right)=k_{m} u, \\
& \quad\left(m_{r}+m_{t}\right) \ddot{x}-m_{r} e \ddot{\alpha} \sin (\alpha)-\left(m_{r}+m_{t}\right) \dot{\omega}_{u} y
\end{aligned}
$$

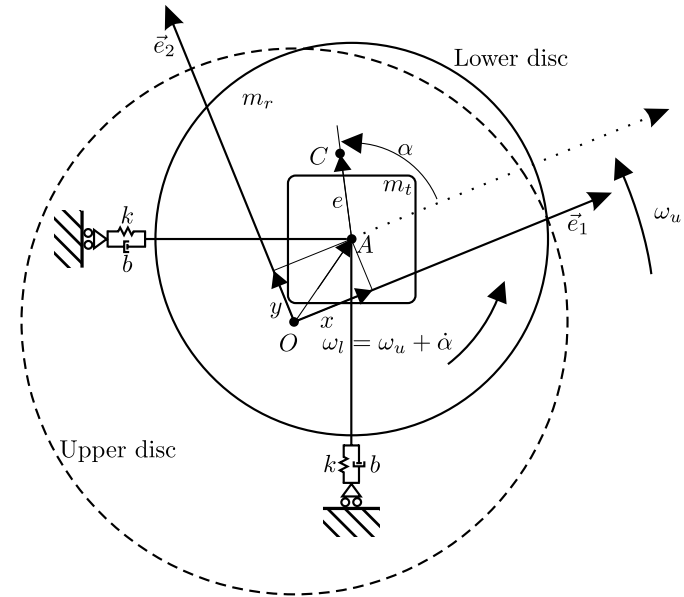

(b) The lower part of the system and the co-rotating coordinate frame $\left\{\vec{e}_{1}, \vec{e}_{2}\right\}$.

$$
\begin{aligned}
& -m_{r} e \dot{\omega}_{u} \sin (\alpha)+b \dot{x}-2\left(m_{r}+m_{t}\right) \omega_{u} \dot{y} \\
& -2 m_{r} e \omega_{u} \dot{\alpha} \cos (\alpha)-m_{r} e \dot{\alpha}^{2} \cos (\alpha)+k x \\
& -\left(m_{r}+m_{t}\right) \omega_{u}^{2} x-b \omega_{u} y-m_{r} e \omega_{u}^{2} \cos (\alpha)=0, \\
& \left(m_{r}+m_{t}\right) \ddot{y}+m_{r} e \ddot{\alpha} \cos (\alpha)+\left(m_{r}+m_{t}\right) \dot{\omega}_{u} x \\
& +m_{r} e \dot{\omega}_{u} \cos (\alpha)+b \dot{y}+2\left(m_{r}+m_{t}\right) \omega_{u} \dot{x} \\
& -2 m_{r} e \omega_{u} \dot{\alpha} \sin (\alpha)-m_{r} e \dot{\alpha}^{2} \sin (\alpha)+k y \\
& -\left(m_{r}+m_{t}\right) \omega_{u}^{2} y+b \omega_{u} x-m_{r} e \omega_{u}^{2} \sin (\alpha)=0, \\
& -m_{r} \ddot{x} e \sin (\alpha)+m_{r} \ddot{y} e \cos (\alpha)+\left(m_{r} e^{2}+J_{C}\right)\left(\ddot{\alpha}+\dot{\omega}_{u}\right) \\
& +m_{r} \dot{\omega}_{u} e(x \cos (\alpha)+y \sin (\alpha))+2 m_{r} e \dot{x} \omega_{u} \cos (\alpha) \\
& +2 m_{r} e \dot{y} \omega_{u} \sin (\alpha) \\
& +T_{f l}\left(\omega_{u}+\dot{\alpha}\right)+m_{r} x \text { e } \omega_{u}^{2} \sin (\alpha) \\
& -m_{r} \text { ye } \omega_{u}^{2} \cos (\alpha)+k_{\theta} \alpha=0 \text {, } \\
& T_{f u}\left(\omega_{u}\right) \in\left\{\begin{array}{lr}
T_{c u}\left(\omega_{u}\right) \operatorname{sgn}\left(\omega_{u}\right), & \text { for } \omega_{u} \neq 0, \\
{\left[-T_{s u}+\Delta T_{s u}, T_{s u}+\Delta T_{s u}\right],} & \text { for } \omega_{u}=0,
\end{array}\right. \\
& T_{c u}\left(\omega_{u}\right)=T_{s u}+\Delta T_{s u} \operatorname{sgn}\left(\omega_{u}\right)+b_{u}\left|\omega_{u}\right|+\Delta b_{u} \omega_{u}, \\
& T_{f l}\left(\omega_{l}\right) \in \begin{cases}T_{c l}\left(\omega_{l}\right) \operatorname{sgn}\left(\omega_{l}\right), & \text { for } \omega_{l} \neq 0, \\
{\left[-T_{s l}, T_{s l}\right],} & \text { for } \omega_{l}=0,\end{cases} \\
& T_{c l}\left(\omega_{l}\right)=T_{c l}+\left(T_{s l}-T_{c l}\right) e^{-\left|\omega_{l} / \omega_{s l}\right|_{s l}^{\delta_{l}}}+b_{l}\left|\omega_{l}\right|, \\
& \omega_{l}=\omega_{u}+\dot{\alpha} .
\end{aligned}
$$


Table 1 Description and estimated values of the parameters of the setup

\begin{tabular}{|c|c|c|}
\hline Parameter & Description & Estimated value \\
\hline$J_{u}\left(\mathrm{~kg} \mathrm{~m}^{2}\right)$ & Moment of inertia of the upper disc with respect to its centre of mass & 0.4765 \\
\hline$k_{m}(\mathrm{Nm} / \mathrm{V})$ & Motor constant & 4.3228 \\
\hline$T_{s u}, \Delta T_{s u}(\mathrm{Nm})$ & $\begin{array}{l}T_{s u}+\Delta T_{s u} \text { and }-T_{s u}+\Delta T_{s u} \text { are the maximum and minimum value of } T_{f u}\left(\omega_{u}\right) \\
\quad \text { for } \omega_{u}=0\end{array}$ & $0.37975,-0.00575$ \\
\hline $\begin{array}{l}b_{u}, \Delta b_{u} \\
\left(\mathrm{~kg} \mathrm{~m}^{2} / \mathrm{rads}\right)\end{array}$ & $\begin{array}{l}b_{u}+\Delta b_{u} \text { and } b_{u}-\Delta b_{u} \text { are the viscous friction coefficients for positive and } \\
\text { negative velocity } \omega_{u}\end{array}$ & $2.4245,-0.0084$ \\
\hline$k_{\theta}(\mathrm{Nm} / \mathrm{rad})$ & Torsional stiffness coefficient & 0.0775 \\
\hline$J_{C}\left(\mathrm{~kg} \mathrm{~m}^{2}\right)$ & Moment of inertia of the lower disc with respect to its centre of mass & 0.0412 \\
\hline$e(\mathrm{~m})$ & Distance between the centre of mass of the lower disc and its geometric centre & 0.00489 \\
\hline$m_{r}(\mathrm{~kg})$ & $\begin{array}{l}\text { Mass of all parts of the lower part of the setup that can rotate around the } \\
\text { geometric centre of the disc }\end{array}$ & 9.9137 \\
\hline$m_{t}(\mathrm{~kg})$ & $\begin{array}{l}\text { Mass of all parts of the lower part of the setup, which do not rotate around the } \\
\text { centre of the disc but move in the same ( } x \text { or } y \text { ) direction during motion of the } \\
\text { lower disc (i.e. one constraint, the brake, the oil box, the upper bearing } \\
\text { housing, the lower bearing housing and the encoder at the lower disc) }\end{array}$ & 3.3202 \\
\hline$k(\mathrm{~N} / \mathrm{m})$ & Bending stiffness coefficient in lateral direction & 2974.25 \\
\hline$b(\mathrm{~N} \mathrm{~s} / \mathrm{m})$ & Damping coefficient in lateral direction & 25 \\
\hline$T_{s l}(\mathrm{~N} \mathrm{~m})$ & Static friction torque at the lower disc $\left(T_{f l}\left(\omega_{l}\right)\right)$ & 0.2781 \\
\hline$T_{c l}(\mathrm{Nm})$ & Coulomb friction torque in $T_{f l}\left(\omega_{l}\right)$ & 0.0473 \\
\hline$\omega_{s l}(\mathrm{rad} / \mathrm{s})$ & Stribeck velocity in $T_{f l}\left(\omega_{l}\right)$ & 1.4302 \\
\hline$\delta_{s l}$ & Stribeck shape parameter at $T_{f l}\left(\omega_{l}\right)$ & 2.0575 \\
\hline$b_{l}\left(\mathrm{~kg} \mathrm{~m}^{2} / \mathrm{rads}\right)$ & Viscous friction coefficient at the lower disc & 0.0105 \\
\hline
\end{tabular}

A description of all symbols and variables is presented in Table 1. A detailed derivation of the dynamic model (1) is given in [23]. In (1), $T_{f u}\left(\omega_{u}\right)$ represents the friction torque at the upper disc caused by friction in the bearings of the upper disc and by the electro-magnetic effect in the DC-motor. Furthermore, $T_{f l}\left(\omega_{l}\right)$ is the friction torque at the lower disc and it comprises the friction in the bearings of the lower disc and the friction induced by the brake mechanism. It can also be seen that the friction torques $T_{f u}\left(\omega_{u}\right)$ and $T_{f l}\left(\omega_{l}\right)$ are modelled using set-valued force laws. Consequently, the model of the system represents a set of differential inclusions.

\subsection{Parameter estimation}

In order to analyze the dynamics of the experimental setup, we need to estimate the parameters of a nonlinear model (1). Parameter estimation is performed using a nonlinear least-squares technique [10]. Since good starting values are needed for the parameters of the setup, we perform the estimation procedure in the following way:
1. We disconnect the upper disc from the lower disc and estimate the parameters of the upper part of the setup $\left(k_{m}, J_{u}\right)$ and the parameters of the friction torque $T_{f u}\left(\omega_{u}\right)\left(T_{s u}, \Delta T_{s u}, b_{u}, \Delta b_{u}\right)$.

2. We connect the upper and lower discs, fix the lower disc in order to avoid motion in lateral direction, and do not apply any normal force at the brake at the lower part of the setup. Then, we estimate the stiffness parameter of the string $\left(k_{\theta}\right)$ and the moment of inertia of the lower $\operatorname{disc}\left(J_{A}=J_{C}+m_{r} e^{2}\right)$.

3. We apply a normal force of $20.5 \mathrm{~N}$ to the brake at the lower disc, use ondina oil 68 as lubrication fluid and estimate the friction force the lower disc $\left(T_{s l}\right.$, $T_{s l}, \omega_{s l}, \delta_{s l}$ and $\left.b_{l}\right)$.

4. We release the lower disc-so it can move in the lateral direction-and estimate the remaining parameters of the model $\left(e, m_{r}, m_{t}, k\right.$ and $\left.b\right)$, and based on those estimates, we determine $J_{C}$ using

$J_{C}=J_{A}-e^{2} m_{r}$.

In order to estimate the parameters of the setup using the nonlinear least-squares method, a quasi-random input 


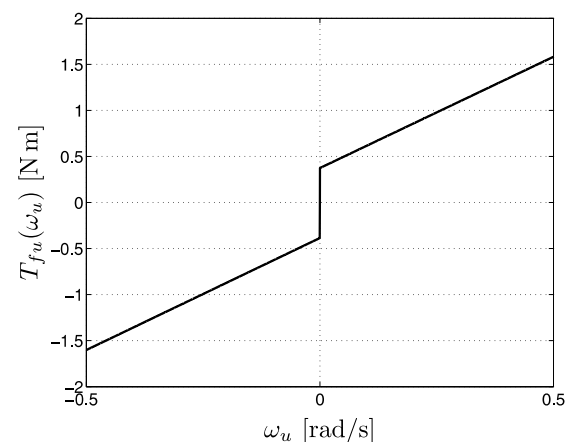

(a) Friction torque at the upper part of the setup.

Fig. 4 Estimated friction models

signal $u$ is used [23]. To objectively asses the accuracy of the estimated parameters, the following $R_{y}^{2}$-criterium is introduced [22]:

$R_{y}^{2}=1-\frac{\sum_{k=1}^{N_{y}}\left(\hat{y}^{(k)}-y^{(k)}\right)^{2}}{\sum_{k=1}^{N_{y}}\left(\hat{y}^{(k)}-\bar{y}\right)^{2}}, \quad \bar{y}=\frac{1}{N_{y}} \sum_{k=1}^{N_{y}} \hat{y}^{(k)}$.

In (3), $y \in \mathbb{R}$ is an output signal of the system. Furthermore, $\hat{y}^{(k)}$ in (3) represents the measured data and $y^{(k)}$ the simulation data when the same input signal is applied both to the system and to model (1). Next, the superscripts in $\hat{y}^{(k)}$ and $y^{(k)}$ indicate the output is a discretized signal obtained at discrete time instants $t_{k}$, $k=1,2, \ldots, N_{y}$. Furthermore, from (3), it can be seen that the maximum value for $R_{y}^{2}$ is 1 . Namely, a value of $R_{y}^{2}$ close to 1 indicates a high quality of the obtained parameter estimates.

Using those steps, the parameters of the model are estimated and the obtained values are given in Table 1. Estimated friction torques are shown in Fig. 4. From Equation (1) and from Fig. 4a, it can be seen that we model the friction at the upper disc as a combination of static friction and viscous friction and that it is asymmetric. Next, from Equation (1) and from Fig. 4b, it can be seen that a negative damping is present at the friction torque at the lower disc for low angular velocities. Validation results, obtained when the quasi-random input signal is applied at the input [23], are given in Fig. 5. The related $R^{2}$ criteria are $R_{\theta_{l}}^{2}=0.9962$ and $R_{r}^{2}=0.8859$, where $r=\sqrt{x^{2}+y^{2}}$ represents the radial displacement of the geometric centre of the lower disc (point $A$ in Fig. 3b). The comparison between

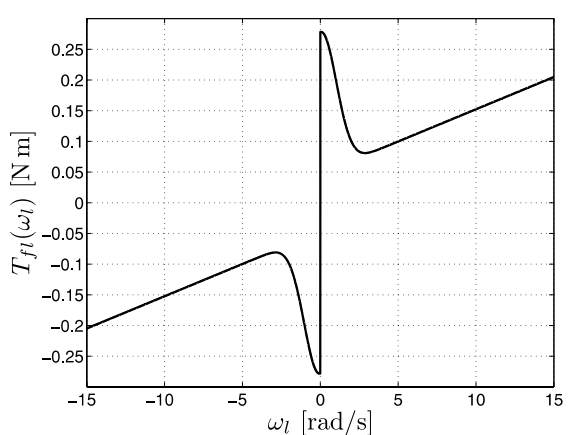

(b) Friction torque at the lower part of the setup.

the responses of the experimental setup and estimated model indicates the high quality of the obtained parameter estimates. A more detailed description of parameter estimation procedure and more validation results (using other input signals) are given in [23].

\section{Analysis of nonlinear dynamic behaviour}

We already noticed that when both the upper and lower discs rotate with a constant angular velocity, a forward whirling motion is performed by the lower disc (lateral vibrations) and this represents an equilibrium point in the co-rotating coordinate frame, in terms of which the model is formulated. Moreover, when torsional vibrations appear in the system, then such motion represents a periodic motion in the co-rotating coordinate frame. Therefore, both the equilibrium points (sets) and the limit cycles of the model as well as the related stability properties are analyzed. Here, we only discuss the results for positive constant input voltages, since the results for negative voltages are qualitatively comparable.

\subsection{Equilibria and related stability analysis}

\subsubsection{Equilibrium points and equilibrium sets}

In the equilibria, the time derivatives of all variables in (1) are zero, i.e. $\dot{\omega}_{u}=\ddot{\alpha}=\dot{\alpha}=\ddot{x}=\dot{x}=$ $\ddot{y}=\dot{y}=0$, for $u=u_{c}$, with $u_{c}$ a constant. Then it holds that $\left(\omega_{u}, \alpha, x, y\right)=\left(\omega_{\mathrm{eq}}, \alpha_{\mathrm{eq}}, x_{\mathrm{eq}}, y_{\mathrm{eq}}\right)$. Furthermore, since $\omega_{l}=\omega_{u}+\dot{\alpha}$, then $\omega_{l}=\omega_{\mathrm{eq}}$ in 


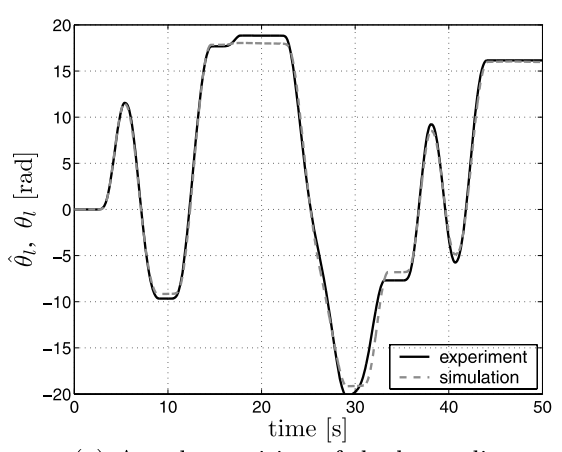

(a) Angular position of the lower disc.

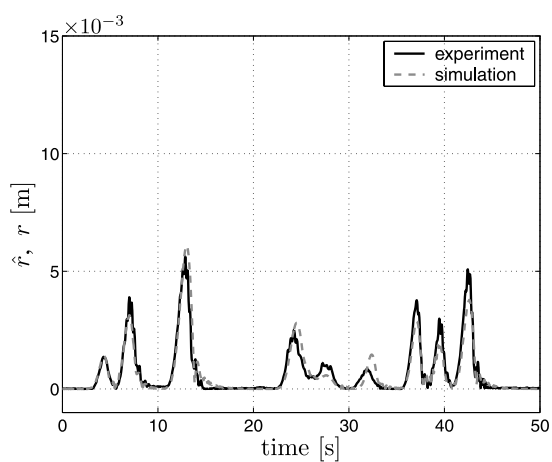

(c) Radial displacement of the geometric center of the lower disc.

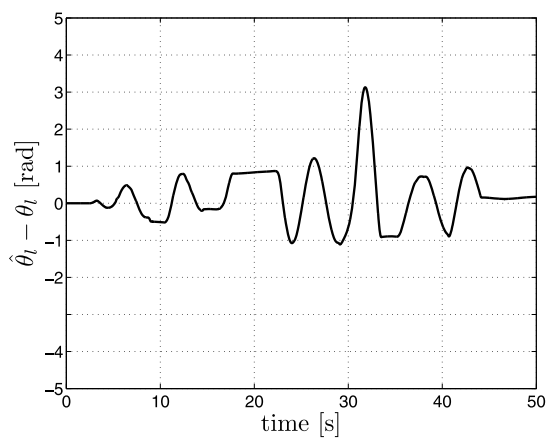

(b) Error between measured $\hat{\theta}_{l}$ and simulated $\theta_{l}$ angular positions.

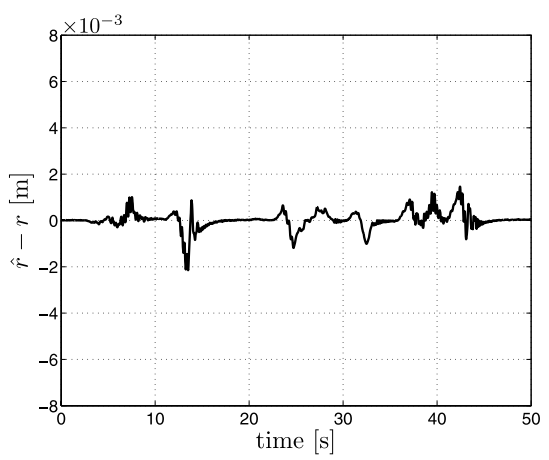

(d) Error between measured $\hat{r}$ and simulated $r$ radial displacements.

Fig. 5 Validation signals in terms of $\theta_{l}$ and $r: R_{\theta_{l}}^{2}=0.9962, R_{r}^{2}=0.8859$

equilibrium. According to (1), in an equilibrium the following holds:

$$
\begin{aligned}
k_{m} u_{c}- & T_{f u}\left(\omega_{\mathrm{eq}}\right)-T_{f l}\left(\omega_{\mathrm{eq}}\right) \\
& -\frac{b e^{2} m_{r}^{2} \omega_{\mathrm{eq}}^{5}}{\left(m_{r}+m_{t}\right)^{2} \omega_{\mathrm{eq}}^{4}+b^{2} \omega_{\mathrm{eq}}^{2}-2 k\left(m_{r}+m_{t}\right) \omega_{\mathrm{eq}}^{2}+k^{2}}=0, \\
\alpha_{\mathrm{eq}}= & -\frac{T_{f l}\left(\omega_{\mathrm{eq}}\right)}{k_{\theta}} \\
& -\frac{b e^{2} m_{r}^{2} \omega_{\mathrm{eq}}^{5}}{k_{\theta}\left(\left(m_{r}+m_{t}\right)^{2} \omega_{\mathrm{eq}}^{4}+b^{2} \omega_{\mathrm{eq}}^{2}-2 k\left(m_{r}+m_{t}\right) \omega_{\mathrm{eq}}^{2}+k^{2}\right)}, \quad(4) \\
x_{\mathrm{eq}}= & \frac{e m_{r} \omega_{\mathrm{eq}}^{2}\left(k-\left(m_{r}+m_{t}\right) \omega_{\mathrm{eq}}^{2}\right) \cos \left(\alpha_{\mathrm{eq}}\right)+b e m_{r} \omega_{\mathrm{eq}}^{3} \sin \left(\alpha_{\mathrm{eq}}\right)}{\left(m_{r}+m_{t}\right)^{2} \omega_{\mathrm{eq}}^{4}+b^{2} \omega_{\mathrm{eq}}^{2}-2 k\left(m_{r}+m_{t}\right) \omega_{\mathrm{eq}}^{2}+k^{2}}, \\
y_{\mathrm{eq}}= & \frac{e m_{r} \omega_{\mathrm{eq}}^{2}\left(k-\left(m_{r}+m_{t}\right) \omega_{\mathrm{eq}}^{2}\right) \sin \left(\alpha_{\mathrm{eq}}\right)-b e m_{r} \omega_{\mathrm{eq}}^{3} \cos \left(\alpha_{\mathrm{eq}}\right)}{\left(m_{r}+m_{t}\right)^{2} \omega_{\mathrm{eq}}^{4}+b^{2} \omega_{\mathrm{eq}}^{2}-2 k\left(m_{r}+m_{t}\right) \omega_{\mathrm{eq}}^{2}+k^{2}} .
\end{aligned}
$$

Consequently, in order to find equilibria of the system, first $\omega_{\text {eq }}$ should be computed by solving the first nonlinear equation in (4). Next, $\alpha_{\text {eq }}$ can be computed using the second equation, and finally, $x_{\mathrm{eq}}$ and $y_{\mathrm{eq}}$ can be determined using the last two equations of (4). Since friction torques $T_{f u}\left(\omega_{u}\right)$ and $T_{f l}\left(\omega_{l}\right)$ are modelled using set-valued friction models, the first two relations in (4) represent an algebraic inclusions and the following situations should be considered:

- equilibria for $\omega_{\mathrm{eq}}>0$, i.e. both the upper and the lower disc rotate with the same constant angular velocity $\omega_{\text {eq }}$ and

- equilibria for $\omega_{\mathrm{eq}}=0$, i.e. both the upper and the lower disc stand still.

For $\omega_{\mathrm{eq}}>0, T_{f u}\left(\omega_{\mathrm{eq}}\right)=T_{c u}\left(\omega_{\mathrm{eq}}\right)$ and $T_{f l}\left(\omega_{\mathrm{eq}}\right)=$ $T_{c l}\left(\omega_{\mathrm{eq}}\right)$ (see (1)). Consequently, such an equilibrium point satisfies the algebraic equations

$$
\begin{aligned}
& k_{m} u_{c}-\left(T_{s u}+\Delta T_{s u}\right)-\left(b_{u}+\Delta b_{u}\right) \omega_{\mathrm{eq}}-T_{c l}\left(\omega_{\mathrm{eq}}\right) \\
& -\frac{b e^{2} m_{r}^{2} \omega_{\mathrm{eq}}^{5}}{\left(m_{r}+m_{t}\right)^{2} \omega_{\mathrm{eq}}^{4}+b^{2} \omega_{\mathrm{eq}}^{2}-2 k\left(m_{r}+m_{t}\right) \omega_{\mathrm{eq}}^{2}+k^{2}}=0, \\
& \alpha_{\mathrm{eq}}=-\frac{T_{c l}\left(\omega_{\mathrm{eq}}\right)}{k_{\theta}}
\end{aligned}
$$




$$
-\frac{b e^{2} m_{r}^{2} \omega_{\mathrm{eq}}^{5}}{k_{\theta}\left(\left(m_{r}+m_{t}\right)^{2} \omega_{\mathrm{eq}}^{4}+b^{2} \omega_{\mathrm{eq}}^{2}-2 k\left(m_{r}+m_{t}\right) \omega_{\mathrm{eq}}^{2}+k^{2}\right)},
$$

and the last two equations of (4). From (1), the first algebraic equation of (5) and due to the fact that $\omega_{\mathrm{eq}}>0$, it can be concluded that the system has such an equilibrium point when

$u_{c}>u_{\mathcal{E} p}:=\frac{T_{s u}+\Delta T_{s u}+T_{s l}}{k_{m}}$.

In general, the first equation in (5) can have more than one solution. However, if

$$
\begin{aligned}
& -\frac{b e^{2} m_{r}^{2} \omega_{\mathrm{eq}}^{4}\left(\left(m_{r}+m_{t}\right)^{2} \omega_{\mathrm{eq}}^{4}+3 b^{2} \omega_{\mathrm{eq}}^{2}-6 k\left(m_{r}+m_{t}\right) \omega_{\mathrm{eq}}^{2}+5 k^{2}\right)}{\left(\left(m_{r}+m_{t}\right)^{2} \omega_{\mathrm{eq}}^{4}+b^{2} \omega_{\mathrm{eq}}^{2}-2 k\left(m_{r}+m_{t}\right) \omega_{\mathrm{eq}}^{2}+k^{2}\right)^{2}} \\
& -b_{u}-\Delta b_{u}-\left.\frac{\mathrm{d} T_{c l}}{\mathrm{~d} \omega_{l}}\right|_{\omega_{l}=\omega_{\mathrm{eq}}} \leq 0
\end{aligned}
$$

then it has one unique solution. Since condition (7) is satisfied for the estimated model, the system has only one equilibrium point for $u_{c}>u_{\mathcal{E} p}$.

Since, in the setup, both torsional and lateral vibrations appear, we are interested in the angular velocity $\omega_{l}$ and radial displacement $r$ of the lower disc in steady state for different constant input voltages $u_{c}$. As already discussed before, when $u_{c}>u_{\mathcal{E} p}, \omega_{l}$ in steady state can be obtained by solving the first algebraic equation in (5). The corresponding radial displacement of the centre of the lower disc (in equilibrium) can be derived from the third and fourth equation in (4) as

$$
\begin{aligned}
r_{\mathrm{eq}} & =\sqrt{x_{\mathrm{eq}}^{2}+y_{\mathrm{eq}}^{2}} \\
& =\frac{m_{r} e \omega_{\mathrm{eq}}^{2}}{\sqrt{\left(m_{r}+m_{t}\right)^{2} \omega_{\mathrm{eq}}^{4}+b^{2} \omega_{\mathrm{eq}}^{2}-2 k\left(m_{r}+m_{t}\right) \omega_{\mathrm{eq}}^{2}+k^{2}}},
\end{aligned}
$$

since $\quad\left(m_{r}+m_{t}\right)^{2} \omega_{\mathrm{eq}}^{4}+b^{2} \omega_{\mathrm{eq}}^{2}-2 k\left(m_{r}+m_{t}\right) \omega_{\mathrm{eq}}^{2}+$ $k^{2}>0$ for every $\omega_{\mathrm{eq}} \in \mathbb{R}$ for the estimated parameters of the setup. If we analyze expression (8), the following is obtained:

$$
\begin{array}{ll}
r_{\mathrm{eq}}=0, & \text { for } \omega_{\mathrm{eq}}=0, \\
r_{\mathrm{eq}} \rightarrow \frac{m_{r} e}{m_{r}+m_{t}}, & \text { for } \omega_{\mathrm{eq}} \rightarrow \infty .
\end{array}
$$

Moreover, the so-called critical speed $\omega_{c}$ [15], i.e. the angular velocity at which the amplitude of lateral vibrations due to an unbalance at the lower disc reaches a local maximum, is given by:

$\omega_{c}=\sqrt{\frac{2 k^{2}}{2 k\left(m_{r}+m_{t}\right)-b^{2}}}$.

For that angular velocity, radial displacement of the lower disc in steady state is

$r_{c}=\frac{2 k m_{r} e}{b \sqrt{4 k\left(m_{r}+m_{t}\right)-b^{2}}}$,

and the corresponding input voltage is

$$
\begin{aligned}
u_{c c} & =\frac{\left(b_{u}+\Delta b_{u}\right) \omega_{c}+T_{s u}+\Delta T_{s u}+T_{c l}\left(\omega_{c}\right)}{k_{m}} \\
+ & \frac{b e^{2} m_{r}^{2} \omega_{c}^{5}}{k_{m}\left(\left(m_{r}+m_{t}\right)^{2} \omega_{c}^{4}+b^{2} \omega_{c}^{2}-2 k\left(m_{r}+m_{t}\right) \omega_{c}^{2}+k^{2}\right)} .
\end{aligned}
$$

The equilibrium branches, with respect to $\omega_{\text {eq }}$, can be constructed by solving the first algebraic nonlinear equation from (4), for various $u_{c}$. In general, that equation can only be solved numerically. Then based on the solution, we can construct the equilibrium branch with respect to $r_{\mathrm{eq}}$.

For $\omega_{\mathrm{eq}}=0$, equilibrium points are such that $\left(\omega_{\text {eq }}, \alpha_{\text {eq }}, x_{\text {eq }}, y_{\text {eq }}\right) \in \mathcal{E}_{i}$ and such equilibria exist when the input voltage is $0 \leq u_{c} \leq u_{\mathcal{E} p}$, where $\mathcal{E}_{i}$ represents the equilibrium set defined by

$$
\begin{aligned}
\mathcal{E}_{i} & =\left\{\left(\omega_{\mathrm{eq}}, \alpha_{\mathrm{eq}}, x_{\mathrm{eq}}, y_{\mathrm{eq}}\right) \in \mathbb{R}^{4} \mid \omega_{\mathrm{eq}}=x_{\mathrm{eq}}=y_{\mathrm{eq}}=0,\right. \\
\alpha_{\mathrm{eq}} & \left.\in\left[\alpha_{\min }, \alpha_{\mathrm{max}}\right]\right\},
\end{aligned}
$$

where $\alpha_{\min }$ and $\alpha_{\max }$ are defined by

$$
\begin{aligned}
& \alpha_{\min }=\max \left(\frac{-k_{m} u_{c}-\left(T_{s u}-\Delta T_{s u}\right)}{k_{\theta}},-\frac{T_{s l}}{k_{\theta}}\right), \\
& \alpha_{\max }=\min \left(\frac{-k_{m} u_{c}+\left(T_{s u}+\Delta T_{s u}\right)}{k_{\theta}}, \frac{T_{s l}}{k_{\theta}}\right) .
\end{aligned}
$$

In order to obtain local stability conditions for the equilibrium points (for $\omega_{\text {eq }} \neq 0$ ), we can use Lyapunov's indirect method. The method can only be 
applied when $\omega_{\mathrm{eq}}>0$ (i.e. condition (6) should be satisfied). Therefore, the model of the system (1) is linearized around the equilibrium point and the stability of the linear model is analyzed. Furthermore, in order to analyze the stability properties of the equilibrium set (13), Lyapunov's stability theorem can be used (see [23]). The results of such analysis are shown in the sequel.

\subsubsection{Bifurcation diagram (nominal case)}

Since, in the setup, both torsional and lateral vibrations appear, we are interested in the angular velocity $\omega_{l}$ and radial displacement $r$ of the lower disc in steady state for different constant input voltages $u_{c}$. More specifically, two bifurcation diagrams (for $\omega_{l}$ and $r$ ) are constructed, with $u_{c}$ as a bifurcation parameter for the estimated parameters given in Table 1. The equilibria are discussed in the previous section. Limit cycles are obtained numerically using a path following technique in combination with a shooting method $[1,27]$ for the estimated model of the system. Herein, the so-called switch model [17] is used to properly deal with the discontinuities in the dynamics, related to the set-valued nature of the friction models.

The results of an extensive bifurcation analysis are shown in the bifurcation diagrams in Figs. 6 and 7. In these figures, the maximal and minimal values of $\omega_{l}$ and $r$ are plotted when a limit cycle is found. The Floquet multipliers, corresponding to these limit cycles, are computed numerically and used to determine the local stability properties of these limit cycles. With respect to the obtained results, the following remarks can be made:

- For $0<u_{c} \leq u_{\mathcal{E} p}$, with $u_{\mathcal{E} p}$ given by (6) (point $A$ in Fig. 7), the system in steady state is in the stick phase, i.e. the system has a locally asymptotically stable equilibrium set described by (13) and (14) (equilibrium branch $e_{1}$ in Fig. 7) (see [23] for a detailed stability argument).

- For $u_{c}=u_{\mathcal{E} p}$ (point $A$ in the bifurcation diagrams), the locally asymptotically stable equilibrium set (13) reduces to a locally asymptotically stable isolated equilibrium point and no change of stability properties occurs. The system has a unique equilibrium point for $u_{c}>u_{\mathcal{E} p}$ since condition (7) is satisfied. Moreover, according to the local stability analysis, a locally asymptotically stable equilibrium branch $e_{2}$ appears (Fig. 7), for which $\omega_{\mathrm{eq}}$ and $r_{\mathrm{eq}}$ increase for increasing $u_{c}$.

- From bifurcation point $B$, an unstable equilibrium branch $e_{3}$ and an unstable periodic branch $p_{1}$ arises (see Fig. 7). At that point, a pair of complex conjugate eigenvalues, related to the linearization of the nonlinear dynamics of (1) around the equilibrium point, cross the imaginary axis. Therefore, a Hopf bifurcation occurs at this point. Point $B$ represents a smooth subcritical Hopf bifurcation point [12, 29] because the unstable periodic branch $p_{1}$ consists of limit cycles without stick-slip.

- The unstable periodic branch $p_{1}$ occurs for input voltages which are smaller than input voltages corresponding to the point $B$. The branch $p_{1}$ is connected to a locally stable periodic branch $p_{2}$ at the point $D$ ( $u_{c}$ at point $D$ is smaller than $u_{c}$ at the point $B$ ), which represents a discontinuous fold bifurcation point, since the periodic branch $p_{2}$ consists of stable limit cycles which represent torsional vibrations with stick-slip (see Fig. 7a). Moreover, a Floquet multiplier crosses through the point +1 in the complex plane.

- For some higher constant input voltage $u_{c}$ (point $E$ in Fig. 6), the locally stable periodic branch $p_{2}$ loses stability and an unstable periodic branch appears (periodic branch $p_{3}$ in Fig. 6) through another discontinuous fold bifurcation (point $E$ in Fig. 6).

- The unstable periodic branch $p_{3}$ is connected to the unstable equilibrium branch $e_{3}$ and the stable equilibrium branch $e_{4}$ in the smooth subcritical Hopf bifurcation point $C$. At that point, a pair of complex conjugate eigenvalues, of the linearized model, cross the imaginary axis.

- For input voltages $u_{c}$ higher than that at point $E$, the asymptotically stable equilibrium branch continues. For increasing $u_{c}$, the steady-state velocity at the lower disc $\omega_{l}$ increases. Note that for such high angular velocities, viscous friction is dominant in the friction at the lower disc (see the estimated friction torque $T_{f l}\left(\omega_{l}\right)$ in Fig. $\left.4 \mathrm{~b}\right)$, which induces the local stability of the equilibrium branch $e_{4}$.

The bifurcation diagram shown in Fig. 6 also shows various branches of steady-state solutions for input voltages $u_{c}>5 \mathrm{~V}$, which is, in fact, outside the working region of the experimental setup. However, since a rich variety of interesting qualitative 
Fig. 6 Bifurcation diagram of the model of the experimental setup when both torsional and lateral vibrations are present

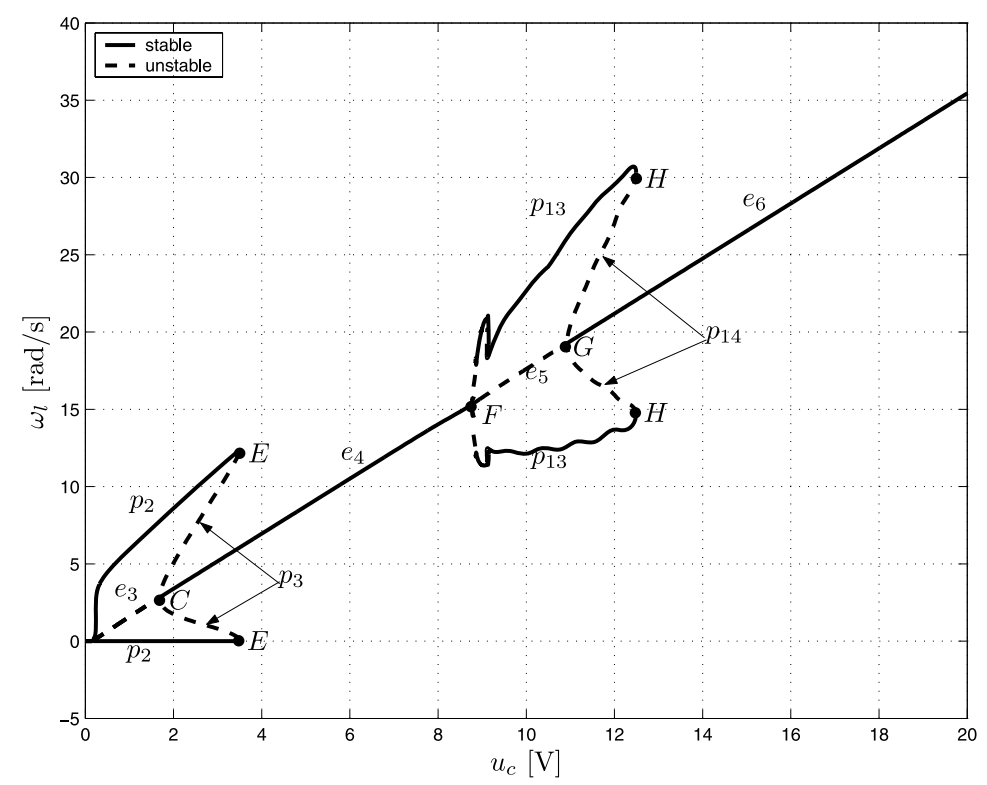

(a) Angular velocity $\omega_{l}$.

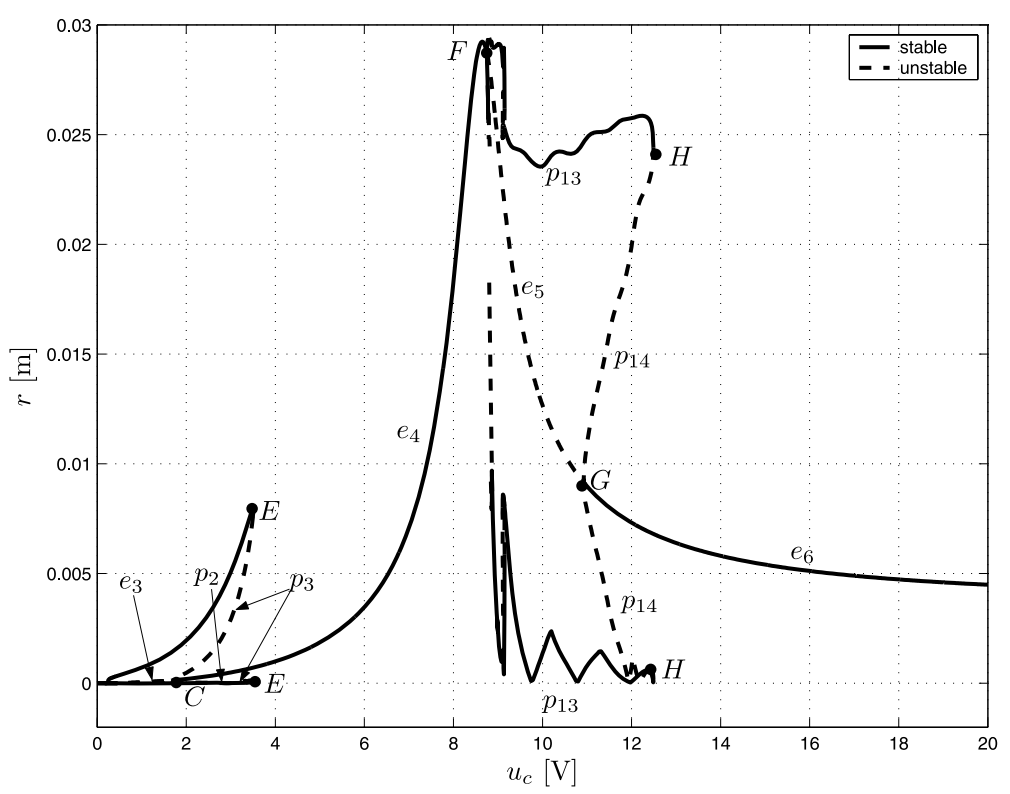

(b) Radial displacement $r$. changes in the dynamic behaviour can appear for those voltages, a detailed bifurcation analysis is provided in Appendix A.1.

The friction-induced vibrations, occurring for $u_{c} \in$ [0 and $3.6 \mathrm{~V}]$ are discussed in more detail in the next section.

\subsection{Friction-induced vibrations}

We have already concluded that the vibrations, which are observed in the model for $u_{c} \in[0$ and $3.6 \mathrm{~V}]$, are induced by friction. Such vibrations are analyzed in more details in [24] when the lower disc is fixed in 


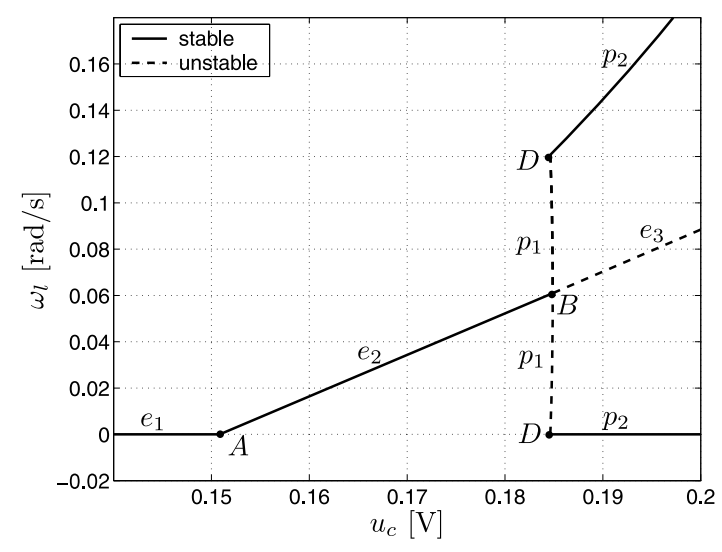

(a) Angular velocity $\omega_{l}$.

Fig. 7 Bifurcation diagram as in Fig. $6 \mathrm{~b}$ for low input voltages

lateral direction. The main cause for lateral vibrations is the presence of the mass-unbalance. Moreover, it appears that this mass-unbalance also affects the friction-induced torsional limit-cycling. Therefore, we analyze the influence of the level of mass-unbalance to the steady-state behaviour of the system for $u_{c} \in$ [0 and $4 \mathrm{~V}]$.

Hereto, we add additional mass $\Delta m$ at a distance of $d_{\Delta}=0.1 \mathrm{~m}$ from the centre of the lower disc in the direction of the already existing unbalance. Consequently, the parameters $e, m_{r}$ and $J_{C}$ of the estimated model (see Table 1) are changed and the new related parameters $e_{\Delta}, m_{r \Delta}$ and $J_{C \Delta}$ are as follows:

$$
\begin{aligned}
e_{\Delta} & =\frac{m_{r} e+d_{\Delta} \Delta m}{m_{r}+\Delta m}, \\
m_{r \Delta} & =m_{r}+\Delta m, \\
J_{C \Delta} & =J_{C}+d_{\Delta}^{2} \Delta m .
\end{aligned}
$$

In Fig. 8, bifurcation diagrams are shown for the estimated system (light-grey line), for $\Delta m=5 \mathrm{~kg}$ (darkgrey line) and $\Delta m=50 \mathrm{~kg}$ (black line). Of course, adding an additional mass $\Delta m=50 \mathrm{~kg}$ to the lower disc, with the estimated mass being $m_{r}=9.9137 \mathrm{~kg}$, is practically impossible. However, we analyze that case in order to observe the effect of additional massunbalance to the steady-state behaviour of the setup. According to the obtained results the following can be concluded:

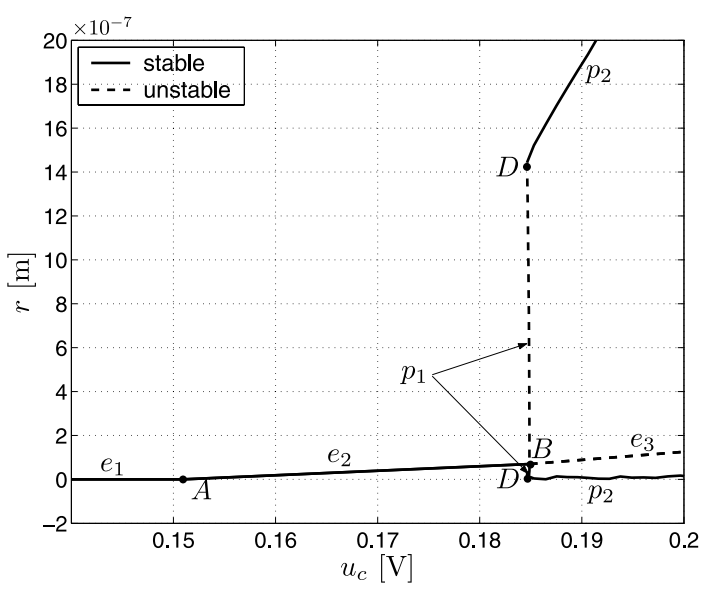

(b) Radial displacement $r$.

- Due to an additional mass-unbalance, the region (in terms of the input voltage) where friction-induced torsional vibrations appear, decreases (see Fig. 8a). Namely, if the mass-unbalance increases, the first fold and Hopf bifurcation points occur at the higher input voltages. Furthermore, the second fold and Hopf bifurcation points occur at significantly lower input voltages (compare the fold bifurcation points $E^{\prime}$ and $E^{\prime \prime}$, and the Hopf bifurcations at $C^{\prime}$ and $C^{\prime \prime}$ in Fig. 8a). Therefore, the region in which the torsional friction-induced vibrations can occur is smaller when the mass unbalance is increased. In Fig. 9 we present the position of the first and the second Hopf bifurcations for various levels of the added mass-unbalance, i.e. we show the region, in which unstable equilibria occur for various $\Delta m$ and for $u_{c} \in[0$ and $5 \mathrm{~V}]$. This figure clearly display the influence of the level of mass unbalance on friction induced instabilities in torsional direction.

- From Fig. $8 \mathrm{~b}$ it can be concluded that when the massunbalance increases, the amplitude of lateral vibrations increases both for the input voltages where torsional vibrations occur (compare periodic branch $p_{2}^{\prime}$ with periodic branches $p_{2 a}^{\prime \prime}, p_{2 b}^{\prime \prime}, p_{2 c}^{\prime \prime}$ and $p_{2 d}^{\prime \prime}$ in Fig. $8 \mathrm{~b}$ ) and where no torsional vibrations appear (compare equilibrium branches $e_{4}^{\prime}$ and $e_{4}^{\prime \prime}$ in the same figure).

- In Fig. 8, we see that the periodic branch $p_{2}^{\prime}$, for $\Delta m=5 \mathrm{~kg}$, splits to four branches $p_{2 a}^{\prime \prime}, p_{2 b}^{\prime \prime}, p_{2 c}^{\prime \prime}$ and $p_{2 d}^{\prime \prime}$, for $\Delta m=50 \mathrm{~kg}$. The periodic branches $p_{2 a}^{\prime \prime}$ and $p_{2 c}^{\prime \prime}$ consist of torsional vibrations with stick-slip, the branch $p_{2 d}^{\prime \prime}$ represents torsional vibrations without 
Fig. 8 Bifurcation diagrams for various levels of mass-unbalance for $u_{c} \in[0$ and $5 \mathrm{~V}]$

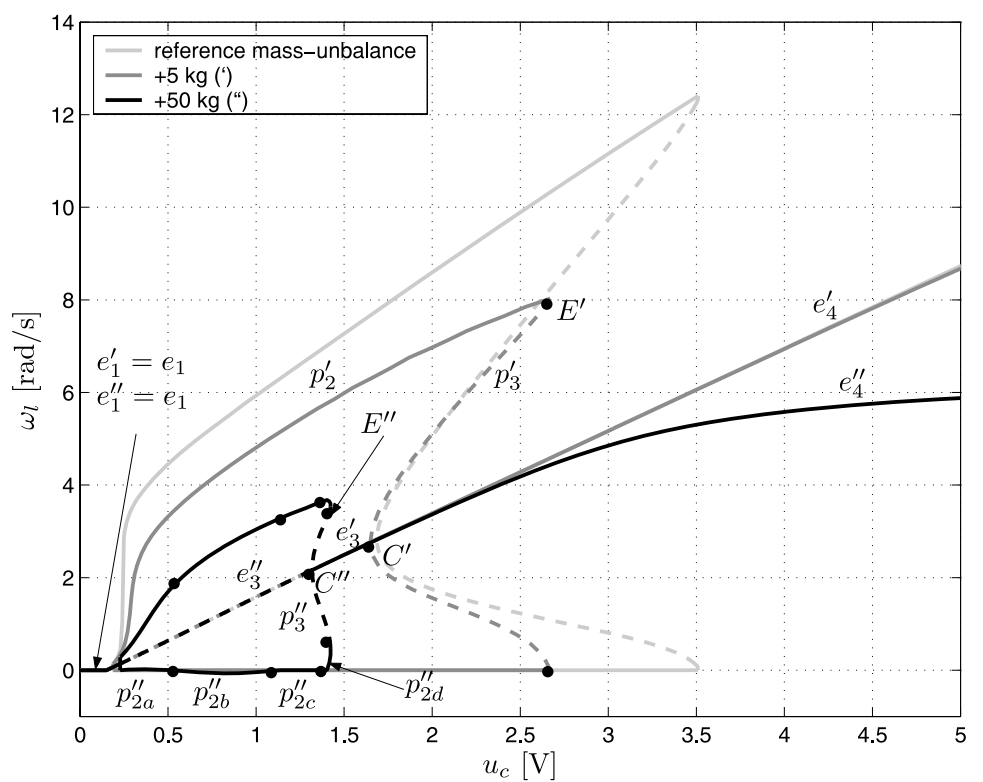

(a) Angular velocity $\omega_{l}$.

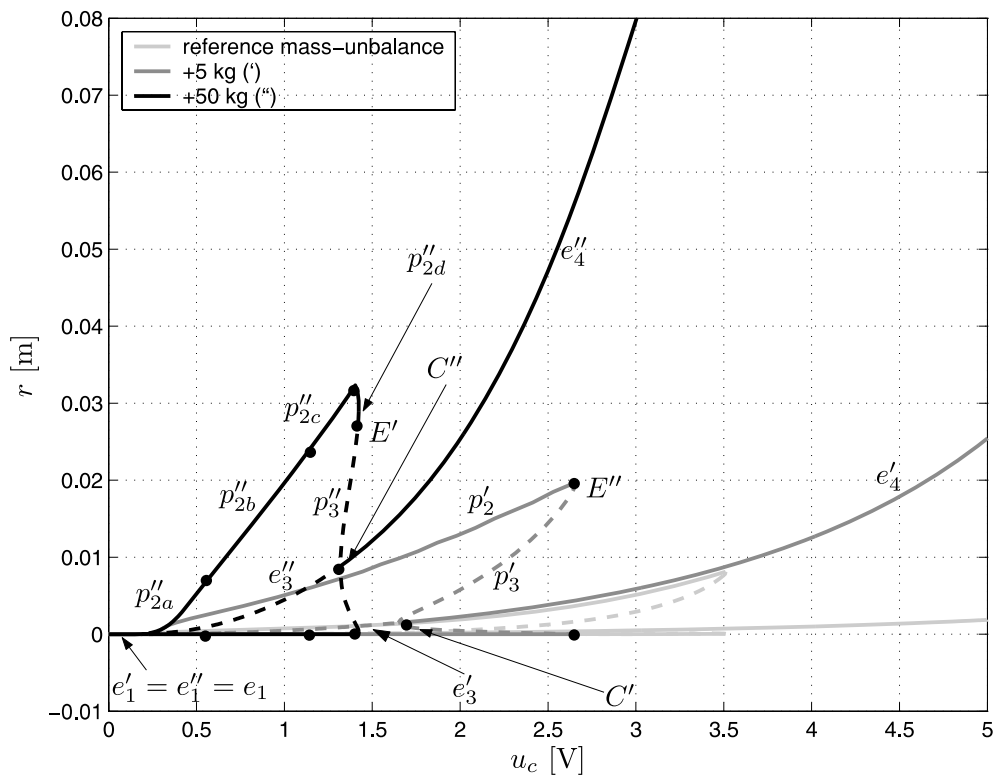

(b) Radial displacement $r$. stick-slip. The branch $p_{2 b}^{\prime \prime}$ represents torsional vibrations where the lower disc starts to rotate in the opposite direction during every period (i.e. $\min \left(\omega_{l}\right)<0$ in a limit-cycle on $p_{2 b}^{\prime \prime}$ ).

- In Fig. 8a, we notice that for $\Delta m=50 \mathrm{~kg}, \partial \omega_{l} / \partial u_{c}$ decreases in steady state for increasing $u_{c}$, see the equilibrium branch $e_{4}^{\prime \prime}$. In Appendix A.2, we analyze that phenomenon when addressing the vibra- tions which occur at higher input voltages $\left(u_{c}>5 \mathrm{~V}\right)$ and are purely due to the coupling between the torsional and lateral modes of vibration in the system.

The effect of the decrease of the friction-induced torsional vibrations when the mass-unbalance is increased can be explained in the following way. When no mass-unbalance is present at the lower disc, the range 
in which friction-induced torsional vibrations can occur is determined by a subtle balance between negative damping at lower velocities and viscous friction at higher velocities (see [23]). Namely, the energy which is released due to the negative damping in the friction characteristics at the lower disc is mainly transformed to the kinetic energy at the lower disc (i.e. $\omega_{l}$ ) and to the potential energy in the low-stiffness string (i.e. $\alpha$ ) and torsional vibrations occur. When mass-unbalance is present at the lower disc, then the energy released due to the negative damping is also transformed to the potential energy stored in the leaf springs and rods (i.e. $r$ ) and kinetic energy related to the translational motion of the lower disc in lateral direction. Consequently, less energy is transformed to kinetic energy of the lower disc in torsional direction and torsional vibrations decrease. In this respect, it is important to notice that, when the level of mass-unbalance is higher, the lateral vibrations increase for angular velocities which are lower than the critical angular velocity, and consequently, less energy can be transformed to kinetic energy of the disc in torsional direction. Hence, torsional vibrations decrease further or they even disappear.

\section{Experimental results}

\subsection{Validation of steady-state behaviour of the setup}

The model of the setup, when ondina oil 68 is used as a lubrication fluid, a normal force of a $20.5 \mathrm{~N}$ is applied at the brake and the $x$ - and $y$-constraints are released, is given by (1) and the parameter estimates are given in Table 1. That setup undergoes both torsional and lateral vibrations. As mentioned earlier, the predictive quality of the estimated model in steady state is of great interest. Therefore, a constant voltage is applied at the input of the DC-motor of the setup and each experiment lasted long enough to guarantee that all transient effects have disappeared; the last $50 \mathrm{~s}$ of the angular velocity $\omega_{l}$ and radial displacement signal $r$ are recorded. However, due to the limited voltage range $(u \in[-5$ and $5 \mathrm{~V}])$ we can only observe the friction-induced vibrations in the setup. Some of the obtained results are shown in Fig. 10. In that figure, the experimental signals (solid black line) and the signals obtained using the estimated model (dashed grey line) in steady state are shown for different constant input voltages. The time-series shown in Fig. 10a-c

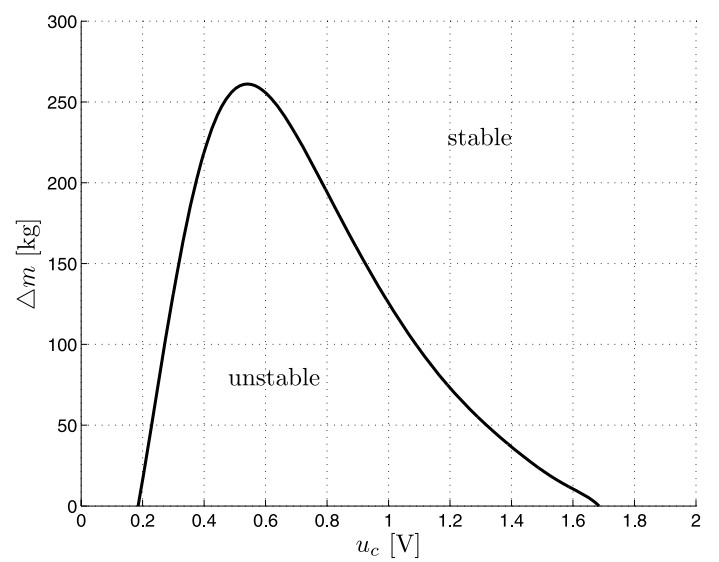

Fig. 9 The regions for which the equilibrium point of the system are locally asymptotically stable and unstable

represent stick-slip limit-cycling (torsional and lateral vibrations) and Fig. 10d represents an equilibrium point (constant velocity at the lower disc and whirling motion of the disc $-r$ is constant). From the comparison between the numerical and experimental results, it can be concluded that with the suggested model, the steadystate behaviour of the setup is modelled accurately.

The same type of bifurcation diagrams, as shown in Fig. 6, are constructed experimentally. However, due to limitations in the DC-motor, the experimental bifurcation diagram is constructed by applying different constant input voltages in the limited voltage range $u_{c} \in[0$ and $5 \mathrm{~V}]$. When no torsional vibrations are observed (as in Fig. 10d), the mean value of the recorded angular velocity and radial displacement are computed and the obtained data are plotted using the symbol "x". Next, when torsional vibrations are observed at the lower disc (as in Fig. 10a-c), the mean values of local maxima and minima of the vibrations are computed. Then, these experimentally obtained data are plotted using the symbol "o". Experimental results, together with the bifurcation diagram obtained by numerical analysis of the estimated model, are shown in Fig. 11a and b. Furthermore, when torsional vibrations are observed in the setup, the period time $T$ of the vibrations is determined as well. The experimental results are compared to the period time of the numerically obtained limit cycles in Fig. 11c. The results, shown in Fig. 11, illustrate the predictive quality of the obtained model.

Both in the numerical and the experimental bifurcation diagram, we recognize the regions which are also 

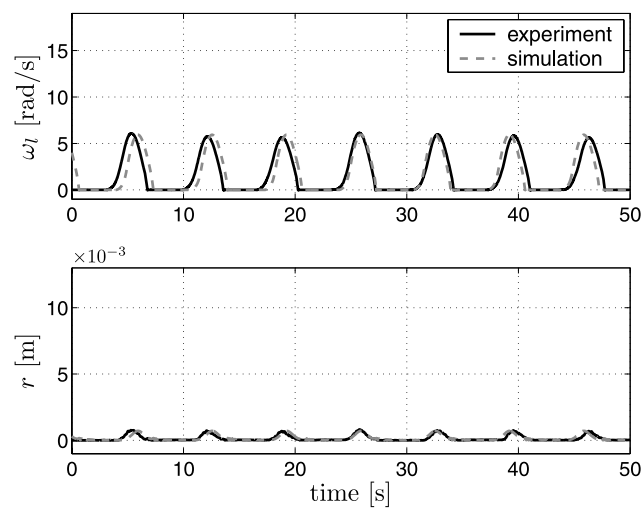

(a) $u_{c}=1.0 \mathrm{~V}$
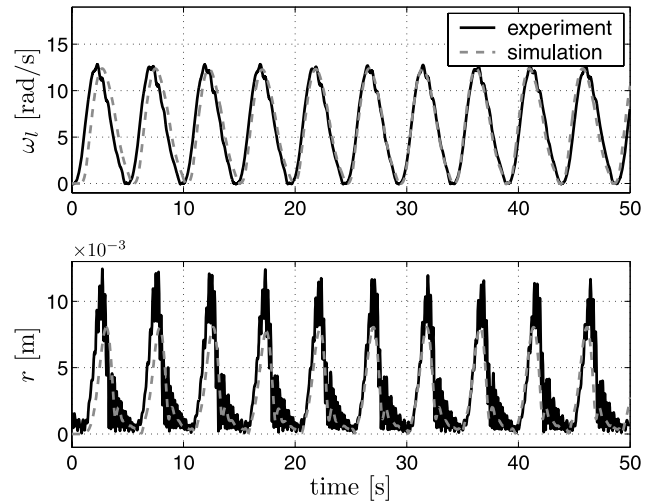

(c) $u_{c}=3.5 \mathrm{~V}$
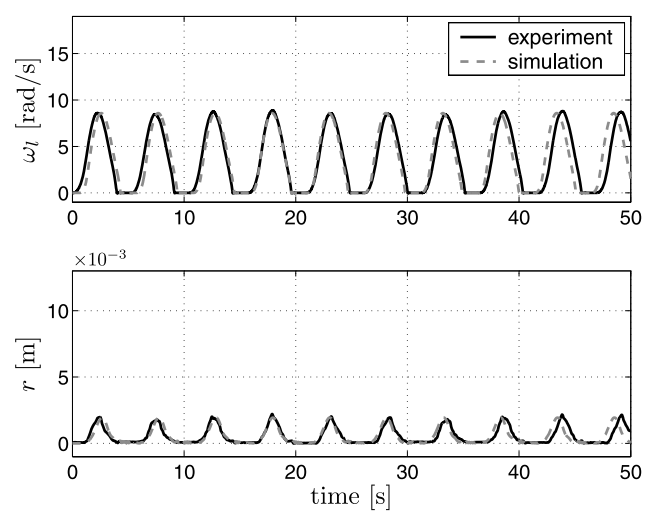

(b) $u_{c}=2.0 \mathrm{~V}$.
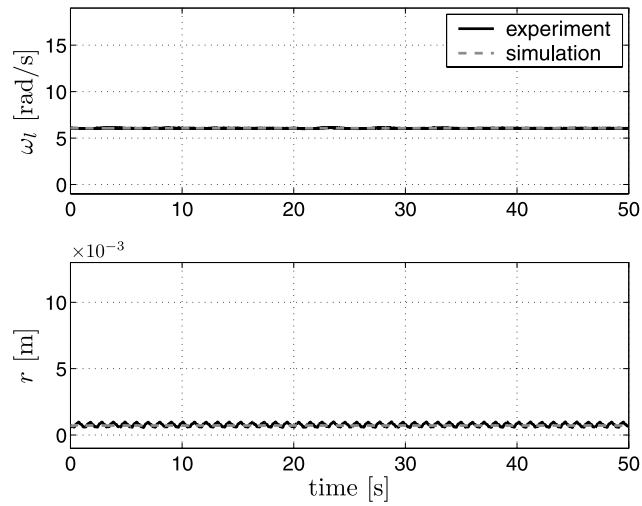

(d) $u_{c}=3.5 \mathrm{~V}$

Fig. 10 Experimental and simulated angular velocity $\left(\omega_{l}\right.$ signal) and radial displacement $(r$ signal) for various constant input voltages and various initial conditions

present when only torsional vibrations are possible in the setup [23, 24]:

- a sticking region, for very low input voltages,

- a region in which only torsional vibrations (i.e. stable limit cycles) appear,

- a region in which torsional vibrations (stable limit cycles) and a constant angular velocity at the lower disc (stable equilibrium points) coexist, and

- a region in which no torsional vibrations can appear in the system in steady state.

For the input voltages $u_{c} \in[3$ and $3.5 \mathrm{~V}]$, we notice that the estimated model is less accurate (see specifically Fig. 11b). The reasons for this fact is that some unmodelled dynamics is present in the setup such as a position-dependent friction at the lower disc $\left(T_{f l}\left(\omega_{l}\right)\right)$, presence of the sticking behaviour in lateral direction due to LVDT displacement sensors (see Fig. 2), anisotropic characteristics of the lower part of the setup in lateral direction. A detailed discussion on unmodelled dynamics in the setup is presented in [23].

\subsubsection{Disappearance of torsional vibrations}

In Fig. 11, with a light-grey line we show the bifurcation diagram of the setup when only torsional and no lateral vibrations are possible, i.e. when $x$ - and $y$ constraints are fixed. If we compare that bifurcation diagram with the bifurcation diagram obtained when lateral vibrations are present in the setup (dark-grey line), we see that the second fold bifurcation point moves towards lower velocities when mass-unbalance and lateral vibrations are present in the system (as predicted in Section 3.2). Namely, when the constraints are fixed, the second fold bifurcation point is observed for $u_{c} \in(3.9$ and $4.0 \mathrm{~V})$ and when the constraints are released the second fold bifurcation point is observed for $u_{c} \in(3.5$ and $3.6 \mathrm{~V})$. 


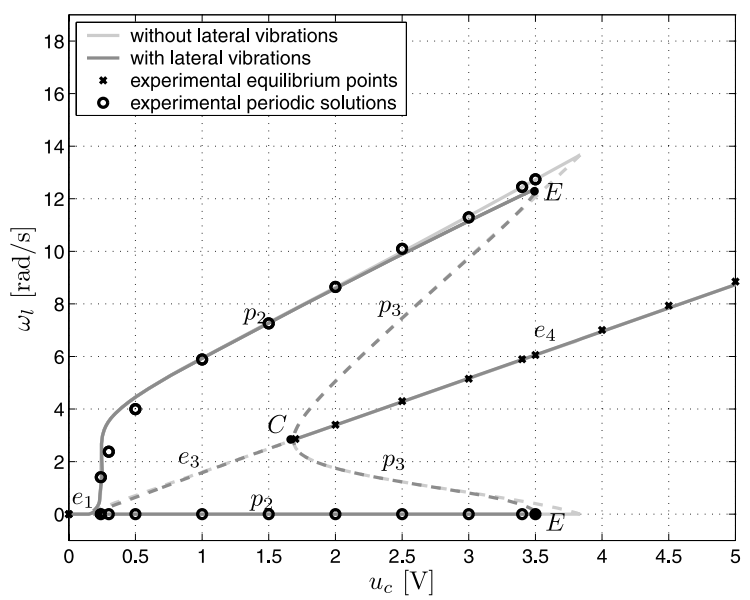

(a) Angular velocity $\omega_{l}$ at the lower disc.

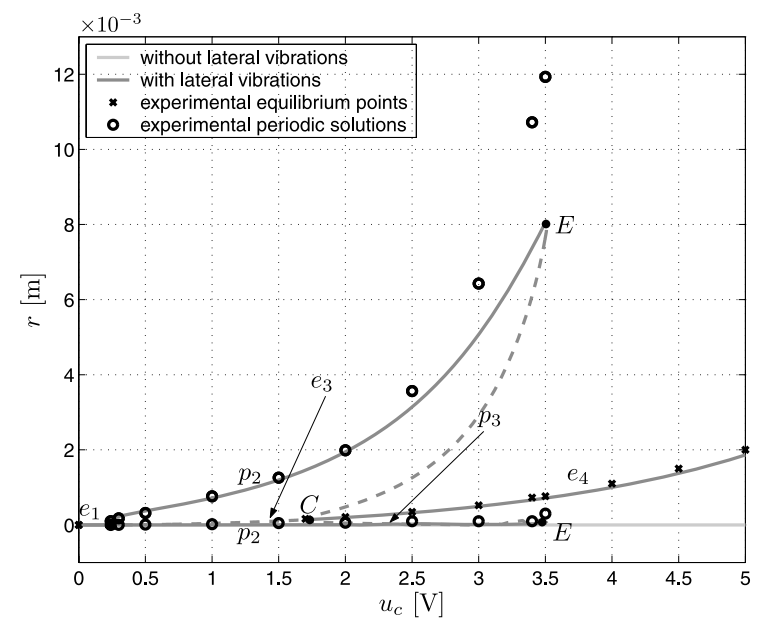

(b) Radial displacement $r$ of the lower disc.

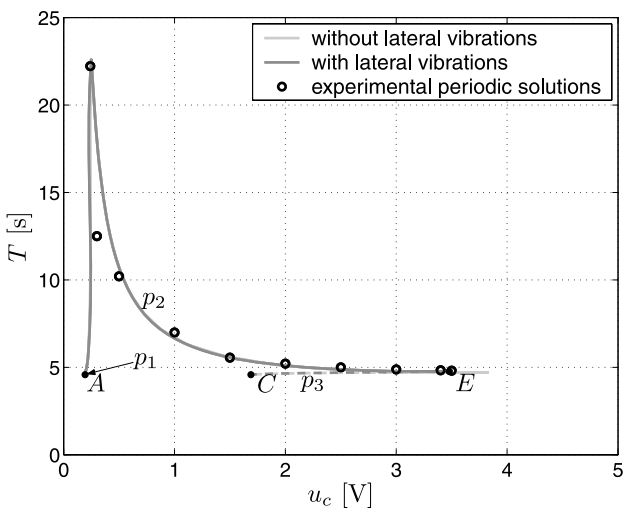

(c) Period time of the periodic solutions.

Fig. 11 Comparison of the numerical and experimental bifurcation diagrams
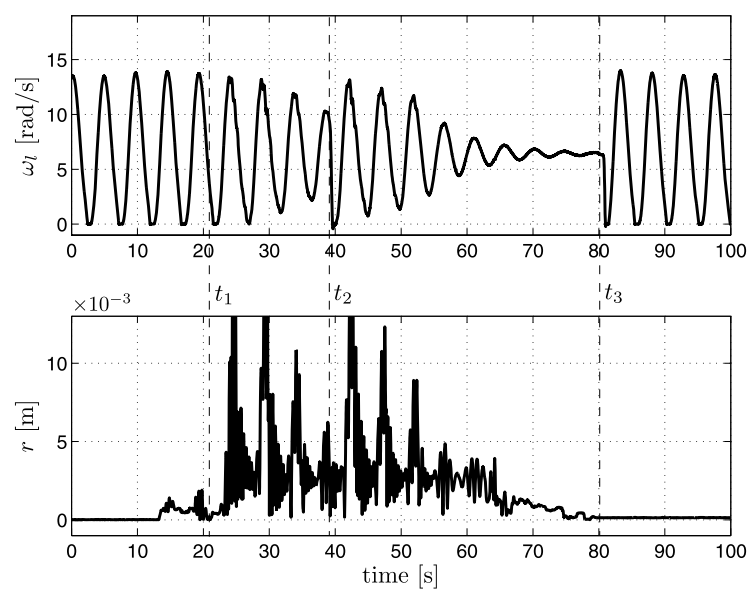

Fig. 12 Indication of disappearance of torsional vibrations when the lower disc moves in lateral direction: experimental results for $u_{c}=3.7 \mathrm{~V}$
In order to show that torsional vibrations can really disappear, for some voltages, due to the existence of lateral vibrations, the following experiment is performed. We fix the constraints, apply a constant input voltage of $u_{c}=3.7 \mathrm{~V}$ and wait long enough to obtain torsional stick-slip vibrations (see Fig. 12). Then, at time instant $t_{1}$, we release the constraints and the lower disc starts to vibrate in lateral direction. After a while, the torsional vibrations disappear even though at time instant $t_{2}$, we tried to induce those vibrations manually, by stopping the lower disc for a very short time in torsional direction. Finally, when we fix again the constraints and stop the lower disc manually (time instant $t_{3}$ in Fig. 12), the system continues with stick-slip vibrations. This experiment provides additional evidence for the fact that torsional vibrations can, indeed, disappear due to the presence of lateral vibrations. In the next section, this 
effect is evidenced quantitatively in both experiments and simulations.

\subsection{Bifurcation analysis for various levels of} mass-unbalance

Since the input voltage which can be applied to the DC-motor is limited $(u \in[0$ and $5 \mathrm{~V}])$, we can only observe the influence of various mass-unbalance to the friction-induced torsional vibrations.

In order to do so, additional masses $\Delta m=$ $0.6032 \mathrm{~kg}$ or $\Delta m=1.2152 \mathrm{~kg}$ are added to the existing mass-unbalance (see Fig. 2) at a distance of approximately $d_{\Delta}=10 \mathrm{~cm}$. Next, for each added mass, no normal force is applied at the brake, the lower disc is fixed using the $x$-and $y$-constraints (shown in Fig. 2). Next,
Fig. 13 Simulated and experimental bifurcation diagrams for various levels of mass-unbalance applied at the lower disc

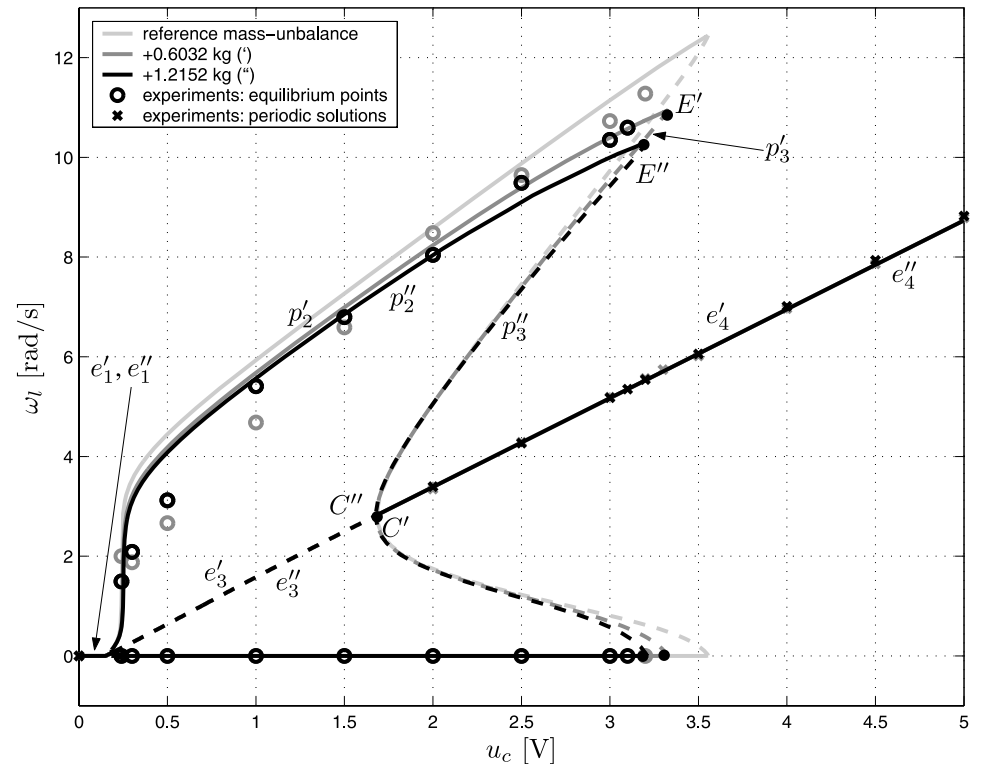

(a) Angular velocity $\omega_{l}$.

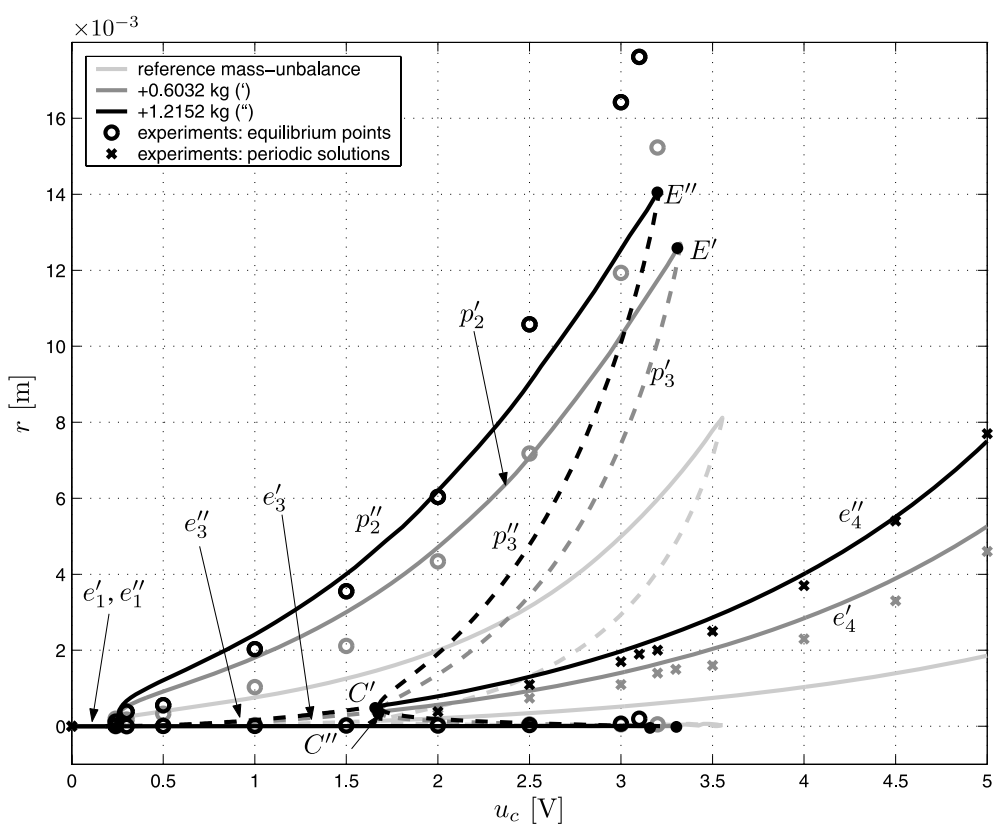

(b) Radial displacement $r$. 
we estimate the distance $d_{\Delta}$ in (15), assuming that all other parameters of the setup are known. In such a way, we obtain that

$$
\begin{array}{ll}
d_{\Delta}=10.85 \mathrm{~cm}, & \text { for } \Delta m=0.6032 \mathrm{~kg}, \\
d_{\Delta}=8.98 \mathrm{~cm}, & \text { for } \Delta m=1.2152 \mathrm{~kg} .
\end{array}
$$

Then, for both mass-unbalances ondina oil 68 is used, a normal force of $20.5 \mathrm{~N}$ is applied, and the model (1), (15) with parameter estimates shown as in Table 1 and Equation (16) is validated. The comparison between the responses of the experimental setup and estimated model indicates the good quality of the obtained parameters for both mass-unbalances.

For each added mass-unbalance, we construct numerical and experimental bifurcation diagrams in the same way as described in Section 4.1 (i.e. when various constant voltages are applied at the input of the DC-motor). The obtained diagrams are shown in Fig. 13.

From those bifurcation diagrams, one can conclude that due to an additional mass-unbalance the region, in which friction-induced torsional vibrations occur (see Fig. 13a), reduces. Namely, for $\Delta m=0.6032 \mathrm{~kg}$, the second fold bifurcation point occurs between $u_{c}=$ $3.2 \mathrm{~V}$ and $u_{c}=3.3 \mathrm{~V}$, and for $\Delta m=1.2152 \mathrm{~kg}$, the fold bifurcation occurs between $u_{c}=3.1 \mathrm{~V}$ and $u_{c}=$ $3.2 \mathrm{~V}$. The same conclusion is derived in Section 3.2, where we discussed the influence of various levels of mass-unbalance to the friction-induced torsional vibrations.

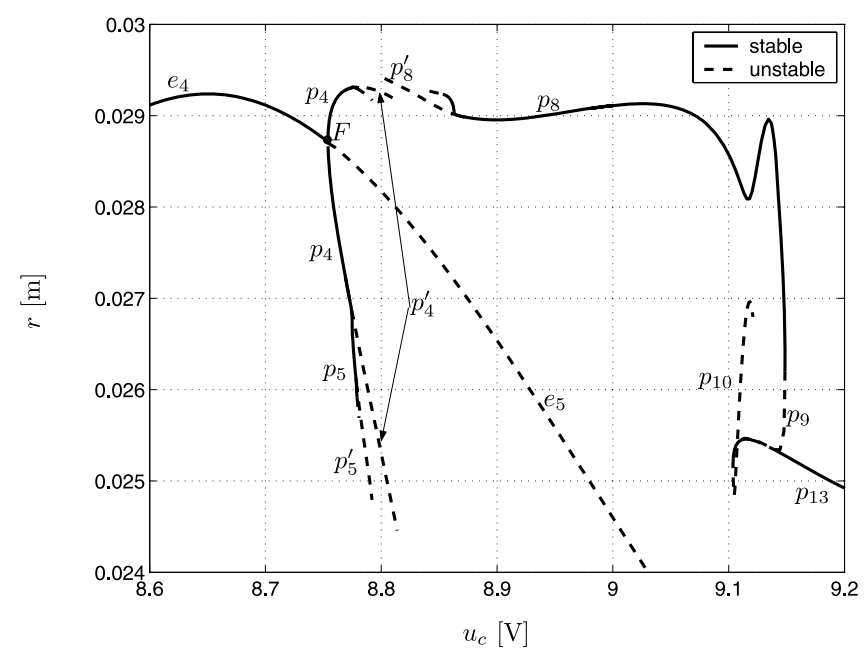

(a) Whole bifurcation diagram, for $u_{c} \in(8.6 \mathrm{~V}, 9.2 \mathrm{~V})$.

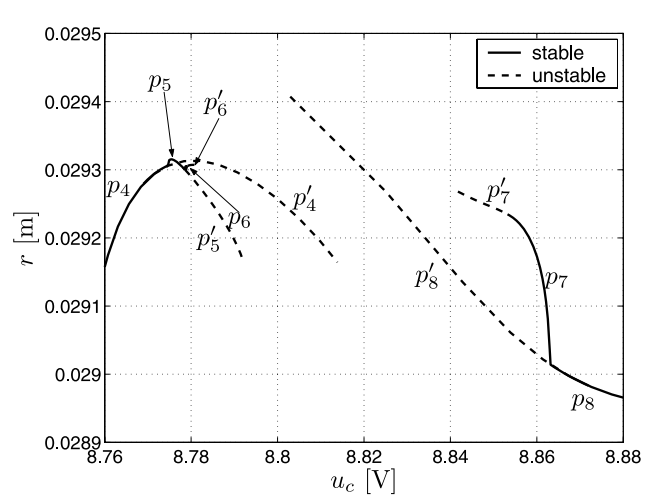

(b) $u_{c} \in(8.76 \mathrm{~V}, 8.88 \mathrm{~V})$.

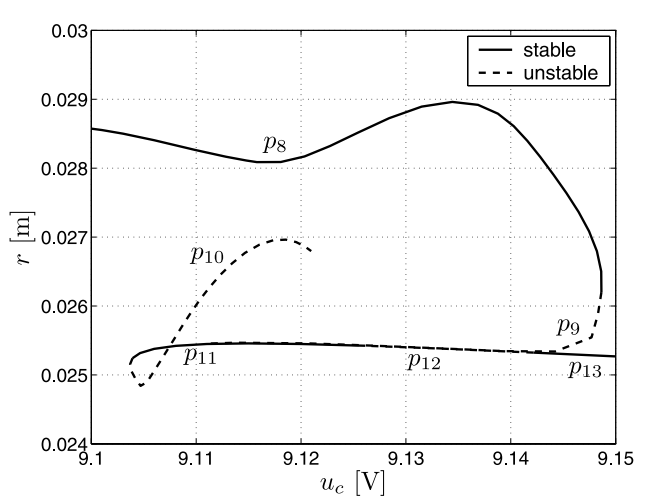

(c) $u_{c} \in(9.1 \mathrm{~V}, 9.15 \mathrm{~V})$.

Fig. 14 Bifurcation diagram as in Fig. $6 \mathrm{~b}$ of radial displacement $r$, for $u_{c} \in(8.6$ and $9.2 \mathrm{~V})$ 


\section{Conclusions}

The aim of this paper is to provide an improved understanding on the interaction between torsional and lateral vibrations in rotor systems with flexibility, massunbalance and dry friction effects. For that purpose, we have analyzed an experimental setup, consisting of two discs interconnected by a low-stiffness string, in which torsional vibrations are induced by friction at the lower disc and lateral vibrations are induced by the presence of a mass-unbalance at the lower disc. However, the results obtained here are relevant for many other engineering systems with friction, unbalance and flexibility. In this context, one can think of drilling systems which are used for exploration of oil and gas, electrical shavers, various turbines, pumps, fans, etc. According to the presented results, the following conclusions are drawn:

- The dynamics of the setup, which is described by differential equations with discontinuous right-hand side (since the friction is modelled with a set-valued force law), is experimentally validated. With these differential inclusions, we successfully modelled equilibrium sets, isolated equilibria and stick-slip limit cycling phenomena (and the related stability properties) which are also observed in the setup. We also observe a discontinuous fold bifurcation both in simulations and experiments. Note that the experimental verification of such nonlinear phenomena, which are explicitly due to the discontinuities in the system, is relatively rare in literature.

- The influence of various levels of mass-unbalance to the steady-state behaviour of the system is studied on a theoretical, numerical and experimental level. Results on all levels confirm that if the level of mass-unbalance increases, the region, in which friction-induced torsional vibrations occur, decreases. Moreover, numerical results show that if the mass-unbalance is high enough, the torsional vibrations can disappear entirely.

- In this class of systems, two types of torsional vibrations can appear:

- friction-induced torsional vibrations,

- torsional vibrations due to a coupling between torsional and lateral dynamics in the system.

Friction-induced vibrations are induced due to a subtle balance between negative damping at low veloc- ities and viscous friction at higher velocities. When the disc rotates with an angular velocity where negative damping is present in the friction, an instability occurs in the system and the lower disc cannot rotate with a constant angular velocity. Consequently, torsional vibrations occur. Moreover, the amplitude of lateral vibrations increases with respect to the amplitude of the lateral vibrations when no torsional vibrations are present in the system.

Torsional vibrations due to coupling between torsional and lateral motions occur for input voltages which are higher than the critical voltage related to the critical angular velocity inducing a resonance in lateral direction. In that working region, torsional vibrations can occur even if no negative damping is present in the friction at the lower disc.

\section{Appendix}

\section{A.1 Bifurcation diagram for $u_{c}>5 \mathrm{~V}$}

When input voltage $u_{c}$ increases from $u_{c}=5 \mathrm{~V}$, the velocity at the lower disc $\omega_{l}$ in steady state continues to increase. For such high angular velocities, viscous friction is dominant in the friction at the lower disc (see the estimated friction torque $T_{f l}\left(\omega_{l}\right)$ in Fig. 4b). However, according to the steady-state analysis, the following periodic solutions are observed in the bifurcation diagrams in Fig. 6 for even higher input voltages:

- At point $F$, the equilibrium point loses stability and an unstable equilibrium branch $e_{5}$ occurs (Fig. 6) as well as a stable periodic branch $p_{4}$ (see Fig. 14a). Point $F$ represents a supercritical Hopf bifurcation point since a pair of complex conjugate eigenvalues, of the linearisation of the nonlinear dynamics of (1) around the equilibrium point, cross the imaginary axis.

- The stable periodic branch $p_{4}$ is connected to an unstable periodic $p_{4}^{\prime}$ via a period doubling bifurcation point $[12,29]$. Consequently, from that point, a stable period-doubled branch $p_{5}$ arises, as shown in Fig. 14a. The periodic branch $p_{5}$ is connected to an unstable periodic branch $p_{5}^{\prime}$, via another period doubling bifurcation point (Fig. 14b). Then, from that point, a stable period-doubled periodic branch $p_{6}$ arises, which is again connected to an unstable branch $p_{6}^{\prime}$ via another period doubling bifurcation 
point (Fig. 14b). The periodic branches $p_{4}-p_{5}-p_{6}$ form a so-called periodic doubling cascade that, according to Strogatz [29], leads to a chaos. If we increase $u_{c}$ even further, unstable periodic branches $p_{7}^{\prime}$ and $p_{8}^{\prime}$ appear. The unstable branch $p_{7}^{\prime}$ is connected to a stable branch $p_{7}$ via a period doubling bifurcation point, as shown in Fig. 14b. The unstable branch $p_{8}^{\prime}$ and the stable period-doubled branch $p_{7}$ are connected to a stable periodic branch $p_{8}$ via another period doubling bifurcation ( $p_{8}$ and $p_{8}^{\prime}$ have the same period time in the bifurcation point). Consequently, $p_{8}-p_{7}$ represents a period doubling cascade which leads to chaos (see [29]). The stable branch $p_{8}$ is connected to an unstable periodic branch $p_{9}$ via a fold bifurcation point (see Fig. 14c). An unstable branch $p_{10}$ is connected to a stable $p_{11}$ branch, via a secondary Hopf bifurcation (Fig. 14c). Branches $p_{10}$ and $p_{11}$ consists of limit cycles which have the same period time in the bifurcation point. The stable branch $p_{11}$ is connected to an unstable $p_{12}$ branch, through a period doubling bifurcation. Then, $p_{12}$ is connected to a stable periodic branch $p_{13}$ via another period doubling bifurcation point. The branches $p_{11}$ and $p_{12}$ have the same period time in the bifurcation point. The same holds for the branches $p_{12}$ and $p_{13}$.

- At the point $H$, the stable periodic branch $p_{13}$ is connected to an unstable branch $p_{14}$ through a fold bifurcation point (Fig. 6).

- The unstable periodic branch $p_{14}$ is connected to the unstable $e_{5}$ and the stable equilibrium branch $e_{6}$ in the subcritical Hopf bifurcation point $G$, i.e. a pair of complex conjugate eigenvalues, related to the linearisation of the nonlinear dynamics of (1) around the equilibrium point, cross the imaginary axis.

Since all periodic branches from $p_{4}$ to $p_{14}$ consist of periodic solutions which do not touch the line $\omega_{l}=0$, all related bifurcation points in that region are smooth bifurcations.

The bifurcation diagrams certainly do not show all periodic branches that exist. For example, not all period-doubled branches are calculated and also other branches may be missing in the bifurcation diagram. However, the presented bifurcation analysis shows that for input voltages outside the working region of the experimental setup, a rich variety of interesting qualitative changes in the dynamic behaviour can appear when the input voltage is changed.
In Appendix A.2, we provide additional insight in the origin of these vibrational phenomena due to the coupling of the torsional and lateral dynamics.

A.2 Vibrations due to coupling between torsional and lateral dynamics

For input voltages $u_{c}>5 \mathrm{~V}$, we observe vibrations which are not induced by friction. Namely, when we remove the negative damping in the friction $T_{f l}\left(\omega_{l}\right)$ and assume that only viscous friction is present at the lower disc, i.e. $T_{f l}\left(\omega_{l}\right)=b_{l} \omega_{l}$, with $b_{l}$ as in Table 1, the torsional friction-induced vibrations disappear for $u_{c} \in[0$ and $5 \mathrm{~V}]$. However, the vibrations for $u_{c}>5 \mathrm{~V}$ are practically unchanged (see [23]).

Due to limitations in the DC-motor, we cannot study torsional vibrations due to the coupling experimentally. Mihajlović [23] has analyzed, numerically, the influence of various levels of mass-unbalance, the stiffness of the low-stiffness string and the damping and stiffness of the construction in lateral direction to the torsional and lateral vibrations which occur due to the coupling between torsional and lateral modes. As a result of this analysis, it is concluded that those torsional vibrations appear almost immediately when the input voltage becomes higher than a so-called critical voltage related to a critical angular velocity $u_{c c}$, given by (12), inducing a resonance in lateral direction $\left(u_{c c}=8.65 \mathrm{~V}\right.$ in Fig. $6 b)$. If we increase the input voltages beyond this resonance, in steady state, the potential energy stored in the leaf springs and rods (related to $r$ ) and the potential energy stored in the low-stiffness spring (related to $-\alpha=\omega_{u}-\omega_{l}$ ) both significantly decrease, despite the fact that extra energy is supplied to the system (due to an increase of $u_{c}$ ). Now, the extra supplied energy is stored in torsional and lateral vibrations (induced by the Hopf bifurcation at point $F$ in Fig. 6. In [23], more detailed discussion can be found about torsional vibrations due to coupling between torsional and lateral dynamics.

Numerical analyses also shows that a higher level of mass-unbalance, a higher stiffness of the string or a higher damping in lateral direction or a lower level of stiffness of the construction in lateral direction decrease the instability region (i.e. decrease the region in which torsional vibrations occur) (see [23]). Moreover, if the levels of all those parameters are such that no torsional vibrations are present in the system, the parameter changes influence in various ways the steady-state 
behaviour of the system. Nevertheless, as a result of the performed analysis in [23], it is concluded that only if the damping in lateral direction increases, both torsional and lateral vibrations decrease which is not the case if we change other parameters.

\section{References}

1. Ascher, U.M., Mattheij, R.M.M., Russell, D.R.: Numerical Solution of Boundary Value Problems for Ordinary Differential Equations. SIAM, Philadelphia (1995)

2. Brett, J.F.: Genesis of bit-induced torsional drill drillstring vibrations. In: Proceedings of the SPE/IADC Drilling Conference, Amsterdam, The Netherlands, pp. 419-428 (1991)

3. Brett, J.F.: Genesis of torsional drillstring vibrations. SPE Drilling Eng. 7(3), 168-174 (1992)

4. Brogliato, B.: Nonsmooth Mechanics. Springer, London (1999)

5. Dankowitz, H., Nordmark, A.B.: On the origin and bifurcations of stick-slip oscillations. Physica D 136(3/4), 280-302 (2000)

6. Di Bernardo, M., Feigin, M.I., Hogan, S.J., Homer, M.E.: Local analysis of $c$-bifurcations in $n$-dimensional piecewisesmooth dynamical systems. Chaos Solut. Fractals 10(11), 1881-1908 (1999)

7. Fritz, R.J.: The effects of an annular fluid on the vibrations of a long rotor. Part 1: Theory. ASME J. Basic Eng. 923-929 (1970)

8. Fritz, R.J.: The effects of an annular fluid on the vibrations of a long rotor. Part 2: Test. ASME J. Basic Eng. 930-937 (1970)

9. Galvanetto, U., Bishop, S.R., Briseghella, L.: Event maps in a stick-slip systems. Nonlinear Dyn. 99-115 (1997)

10. Gelb, A., Kasper, J.F., Nash, R.A., Price, C.F., Sutherland, A.A.: Applied Optimal Estimation. MIT Press, London (1978)

11. Gunter, E.J., Humphris, R.H., Springer, H.: Influence of unbalance on the nonlinear dynamical response and stability of flexible rotor-bearing systems. Rotor Dyn. Instability 115, 37-58 (1983)

12. Khalil, H.K.: Nonlinear Systems. Prentice Hall, Englewood Cliffs, NJ (2000)

13. Krauter, A.I.: Generation of squeal/chatter in waterlubricated elastomeric bearings. ASME J. Lubricat. Tech. 103, 406-413 (1981)

14. Kunze, M., Küpper, T.: Qualitative bifurcation analysis of a non-smooth friction-oscillator model. Z. angewandte Math. Phys. (ZAMP) 48, 87-101 (1997)
15. Lee, C.-W.: Vibration Analysis of Rotors. Kluwer, Dordrecht, The Netherlands (1993)

16. Leine, R.I.: Bifurcations in Discontinuous Mechanical Systems of Filippov-Type. Ph.D. thesis, Eindhoven University of Technology, The Netherlands (2000)

17. Leine, R.I., Nijmeijer, H.: Dynamics and Bifurcations of Non-Smooth Mechanical Systems. Springer, Berlin (2004)

18. Leine, R.I., Van Campen, D.H.: Discontinuous fold bifurcations. Syst. Anal. Model. Simulation 43(3), 321-332 (2003)

19. Leine, R.I., Van Campen, D.H., De Kraker, A., Van den Steen, L.: Stick-slip vibrations induced by alternate friction models. Nonlinear Dyn. 16, 41-54 (1998)

20. Leine, R.I., Van Campen, D.H., Glocker, C.: Nonlinear dynamics and modeling of various wooden toys with impact and friction. J. Vib. Control 5, 25-78 (2003)

21. Leine, R.I., Van Campen, D.H., Keultjes, W.J.G.: Stick-slip whirl interaction in drillstring dynamics. ASME J. Vib. Acoustics 124, 209-220 (2002)

22. Mayers, R.H.: Classical and Modern Regression with Applications. Dixbury Press, Boston (1989)

23. Mihajlović, N.: Torsional and Lateral Vibrations in Flexible Rotor Systems with Friction. Ph.D. thesis, Eindhoven University of Technology, The Netherlands (2005)

24. Mihajlović, N., Van de Wouw, N., Hendriks, M.P.M., Nijmeijer, H.: Friction-induced limit cycling in flexible rotor systems: an experimental drill-string set-up. Nonlinear Dyn. (Special Issue Exp. Anal. Control Nonsmooth Dyn. Syst.: Theory Exp.), in press

25. Mihajlović, N., Van Veggel, A.A., Van de Wouw, N., Nijmeijer, H.: Analysis of friction-induced limit cycling in an experimental drill-string set-up. ASME J. Dyn. Syst. Meas. Control 126(4), 709-720 (2004)

26. Muszynska, A.: Whirl and whip - Rotor/bearing stability problems. J. Sound Vib. 110(3), 443-462 (1986)

27. Parker, T.S., Chua, L.O.: Practical Numerical Algorithms for Chaotic Systems. Springer, Berlin (1989)

28. Popp, K., Stelter, P.: Stick-slip vibrations and chaos. Philos. Trans. R. Soc. London 332, 89-105 (1990)

29. Strogatz, S.H.: Nonlinear Dynamics and Chaos. Westview Press, Cambridge, UK (2000)

30. Tondl, A.: Some Problems of Rotor Dynamics. Chapman and Hall, London (1965)

31. Van de Vorst, E.L.B.: Long Term Dynamics and Stabilization of Nonlinear Mechanical Systems. Ph.D. thesis, Eindhoven University of Technology, The Netherlands (1996)

32. Van de Vorst, E.L.B., Fey, R.H.B., De Kraker, A., Van Campen, D.H.: Steady-state behaviour of flexible rotor dynamics systems with oil journal bearings. In: Proceedings of the WAM of the ASME, Symposium on Nonlinear and Stochastic Dynamics, New York, pp. 107-114 (1994) 